pi-qg-245

Lpt-Orsay-11-99

ICMPA-MPA/2011/017

\title{
A Renormalizable 4-Dimensional Tensor Field Theory
}

\author{
Joseph Ben Geloun ${ }^{a, c, \dagger}$ and Vincent Rivasseau ${ }^{b, \ddagger}$ \\ ${ }^{a}$ Perimeter Institute for Theoretical Physics \\ 31 Caroline St. N., ON, N2L 2Y5, Waterloo, Canada \\ ${ }^{b}$ Laboratoire de Physique Théorique, CNRS UMR 8627 \\ Université Paris-Sud, 91405 Orsay, France \\ ${ }^{c}$ International Chair in Mathematical Physics and Applications \\ (ICMPA-UNESCO Chair), University of Abomey-Calavi, \\ 072B.P.50, Cotonou, Rep. of Benin \\ E-mails: †jbengeloun@perimeterinstitute.ca, ${ }^{\ddagger}$ rivass@th.u-psud.fr
}

\begin{abstract}
We prove that an integrated version of the Gurau colored tensor model supplemented with the usual Bosonic propagator on $U(1)^{4}$ is renormalizable to all orders in perturbation theory. The model is of the type expected for quantization of space-time in $4 D$ Euclidean gravity and is the first example of a renormalizable model of this kind. Its vertex and propagator are four-stranded like in $4 D$ group field theories, but without gauge averaging on the strands. Surprisingly perhaps, the model is of the $\phi^{6}$ rather than of the $\phi^{4}$ type, since two different $\phi^{6}$-type interactions are log-divergent, i.e. marginal in the renormalization group sense. The renormalization proof relies on a multiscale analysis. It identifies all divergent graphs through a power counting theorem. These divergent graphs have internal and external structure of a particular kind called melonic. Melonic graphs dominate the $1 / \mathrm{N}$ expansion of colored tensor models and generalize the planar ribbon graphs of matrix models. A new locality principle is established for this category of graphs which allows to renormalize their divergences through counterterms of the form of the bare Lagrangian interactions. The model also has an unexpected anomalous log-divergent $\left(\int \phi^{2}\right)^{2}$ term, which can be interpreted as the generation of a scalar matter field out of pure gravity.
\end{abstract}

Pacs numbers: 11.10.Gh, 04.60.-m

Key words: Renormalization, tensor models, quantum gravity. 


\section{Introduction}

The standard model is built out of renormalizable 4 dimensional quantum field theories. In the Wilsonian point of view this is natural since these theories have long-lived logarithmic flows. They can survive almost unchanged through long sequences of renormalization group transformations. Our universe seems to favor such theories because it is very large (at least in terms of the Planck scale). It would be desirable to describe also quantum gravity with a similar renormalizable model [1].

However the ordinary approach to quantize the Einstein-Hilbert action around flat space is well known to lead to a perturbatively non-renormalizable theory. Attention has turned to add symmetries and extended objects (supergravity, superstring and related approaches) or to create space-time itself from more fundamental entities. In this second point of view these entities can still obey the rules of a more abstract "pregeometric" quantum field theory but it is natural to drop some of the fundamental axioms, for instance ordinary locality and Poincaré invariance. Let us from now on restrict ourselves to Euclidean quantum field theory. The most natural assumption is that classical Euclidean space-time and general relativity could be an effective product of such a pregeometric quantum field theory resulting from a phase transition, just as hadronic physics is the effective product of QCD.

Until now the main success in this direction is the random matrix approach to the quantization of $2 D$ gravity [2]. It produces indeed a theory of continuous Riemann surfaces through a phase transition with computable critical exponents [3, 4]. It can also reproduce through a double scaling a sum of surfaces of different genera [5, 6, 7]. Moreover the theory allows fruitful applications to $2 D$ statistical physics through the KPZ map [8, 9, 10, 11]. This success relies on a fundamental tool to analyze the statistical properties of large random matrices, namely the $1 / N$ expansion [12.

Random tensor models [13, 14, 15, 16] of rank $D \geq 3$ are the first and most natural attempt to generalize this success to higher dimensions $D \geq 3$. The natural vertex is the $D$-stranded $\phi^{D+1}$-type vertex associated to the complete graph on $D+1$ points. Since such points define a $D$-simplex, the Feynman graphs of the theory are dual to triangulations of $D$-dimensional topological spaces. However until recently there was no way to address statistical properties of large tensors of rank higher than 2 through a $1 / N$ expansion, hence such models have been mostly studied through computer simulations. See also [17, 18, 19] for other related approaches.

Group field theory is a special kind of random tensor model in which one adds a Lie group $G$ and a gauge invariance [20, 21, 22, 23, 24] $]^{1}$. In dimension $D$ the natural Lie group is $S O(D)$ (or its covering group). Gauge invariance consists in averaging over a single simultaneous action of $G$ on all $D$-strands of the propagator ${ }^{2}$. This gauge invariance implements the flatness condition of the $B F$ theory, because it ensures trivial holonomy for

\footnotetext{
${ }^{1}$ Group field theory is related to loop quantum gravity since the Feynman amplitudes of group field theory are the spinfoams in the covariant version of LQG. But it improves the latter with key ingredients: canonical combinatoric weights for the spinfoams from Wick theorem, plus the potential to harness the power of quantum field theory tools: functional integrals, non-perturbative expansions and the renormalization group.

${ }^{2}$ In the initial paper of Boulatov and many subsequent works, the propagator is incorporated into the vertex, an unfortunate convention from the QFT point of view.
} 
parallel transport of vectors along all faces of the triangulated space. In three dimensions it seems related to the quantization of gravity because the classical Einstein-Hilbert action reduces to the $B F$ theory in $D=3$.

In four dimensions more elaborate propagators have been proposed [25, 26, 27, 28] to implement the Plebanski simplicity constraints on the 4 dimensional Ooguri GFT or $4 D B F$ theory. Hopefully, this could free the local modes of classical $4 D$ gravity, those responsible for gravitational waves. However, the analysis of the corresponding amplitudes has turned out to be harder than expected [29]. Detailed studies for the power counting of group field theory amplitudes [30, 31, 32, 33, 34, 35, 36] have not lead to any renormalizable group field theory yet.

Recently a breakthrough occurred. A new class of colored models [37] provided at last tensor theories and group field theories with their missing analytic tool, namely the $1 / N$ expansion [38, 39, 40. Results on statistical mechanics on random $D \geq 3$ geometries followed quickly [41, 42, 43]. We refer to [44] for a review and to [45, 46, 47, 48] for other results or aspects of this thriving subject.

Even more important perhaps, actions for uncolored random tensor theories were developed [49]. They obey an infinite dimensional symmetry algebra, based on $D$-ary trees and their fusion rules, which we propose to call the Gurau algebra. This theory and this symmetry has been proved universal in the precise sense of probability theory: every independentidentically-distributed or even invariant probability law on uncolored random tensors is governed in the large $N$ limit by the $1 / N$ expansion of colored models [50]. Hence it is the correct extension in higher dimensions of the central limit theorem and of the Wigner-Dyson theory of random matrices.

This breakthrough opens a new program, namely the systematic investigation of tensor field theories of rank higher than 2 and the classification of their renormalization group flows and critical points using these new analytic tools. We hope this could lead to a simpler and more convincing quantization of gravity in 3 and 4 dimensions. It could also provide the correct extension to higher dimensions of $2 D$ conformal symmetry and integrability which have been so useful in the study of $2 D$ statistical mechanics models and of their phase transitions.

This paper is a first step in this program. We use the $1 / N$ expansion of colored random tensors to build the first renormalizable uncolored rank 4 tensor quantum field theory. Our model can be considered as a natural higher rank analog of the Grosse-Wulkenhaar model [51, 52], which was built around the ordinary $1 / N$ expansion of random matrices. It can be also considered as a group field theory with group $G=U(1)$ but we prefer not to use this terminology since we perform no gauge averaging on the propagator strands. Our model is a four dimensional quantum field theory of a single scalar field with the ordinary $\left(-\Delta+m^{2}\right)^{-1}$ propagator. For earlier approaches to group field theory with inverse Laplacian propagators see [53] and references therein. We also mention that the requirement of Laplacian dynamics in a renormalization analysis of group field theory has been underlined in [54]. Each coordinate is associated to a tensor index, so the model is both 4 dimensional and rank 4. For simplicity, we choose to formulate the theory on the four dimensional torus $T_{4}=U(1)^{4}$ rather than on $\mathbb{R}^{4}$, but this is not a fundamental feature ${ }^{3}$.

\footnotetext{
${ }^{3}$ The compact four dimensional space $T_{4}=U(1)^{4}$ on which the theory lives could be replaced by $\mathbb{R}^{4}$. This
} 
Only the interaction of our model is new. We obtain this interaction by truncating the infinite series of melonic terms in the Gurau action [49] to eliminate the irrelevant terms. This parallels exactly what is done on the infinite series of local interaction terms $\int \phi(x)^{n} d x$ in ordinary renormalizable quantum field theory. The usual $\int \phi^{4}(x) d x$ action is the correct truncation for renormalizability in 4 dimensions. Irrelevant terms do not appear in the bare action. It is also the recipe for renormalizable matrix-like quantum field theories such as the Grosse-Wulkenhaar model, where the infinite series of $\operatorname{Tr} M^{n}$ terms is truncated (in that model again to order 4). This is because the $\operatorname{Tr} M^{n}$ terms are the right matrix analogs of local interactions. Similarly melonic terms are the right analogs of local interactions for tensor theories of rank 3 or more 4 .

It happens that 6 is the right order of truncation for this model to get just renormalizability with the $\left(-\Delta+m^{2}\right)^{-1}$ propagator. We need also to add the correct terms of order 4 and 2 . The theory generates a single unexpected $\left(\int \phi(x)^{2} d x\right)^{2}$ anomaly which could be interpreted as the generation of a scalar matter field out of pure gravity (see Subsection 6.3). Adding the corresponding fourth order term to the Lagrangian we prove through a multiscale analysis that our model is renormalizable to all orders of perturbation theory.

Our model being defined on a compact space there is only one half-direction for the renormalization group. According to the usual quantum field theory conventions we call it the ultraviolet direction, as it describes short range fluctuations of the field 5 .

Section 2 introduces the model and notations and states our main theorem. Section 3 writes its multiscale decomposition and bounds. Sections 4 and 5 identify the contributions to renormalize, including the anomalous term. Section 6 performs renormalization through suitable Taylor expansions around the local melonic parts of every divergent subgraph in the multiscale analysis. Section 7 lists some perspectives and open problems. An appendix provides some details on calculations invoked in the text and introduces a similar just renormalizable theory in dimension 3. The important physical issues of the underlying model symmetries, renormalization group flow and possible phase transitions of such models are postponed to subsequent works.

\section{The Model}

We start by a blitz review of the basic ingredient, colored rank 4 tensor field theory [37]. Let us consider a family of $5=4+1$ complex fourth rank tensor fields over the group $U(1)$, $\varphi^{a}: U(1)^{4} \rightarrow \mathbb{C}$. They are labeled with an index $a=0,1,2, \ldots, 4$, called color. These

would introduce the usual distinction between infrared and ultraviolet divergences. The infrared divergences could be cured by an infrared regulator such as an harmonic potential à la Grosse-Wulkenhaar. This is left to a future study.

${ }^{4}$ We nevertheless agree that when written in terms of coordinates on $T^{4}$ the melonic interactions look unfamiliar at first sight. The four different coordinates of $U(1)^{4}$ correspond to different strands in the propagator which are identified according to the melonic drawings. The resulting interaction is certainly not local in the usual sense.

${ }^{5}$ Recall that the large Fourier modes in group field theory or spin-foams are often considered the infrared direction, because of a different interpretation. If space-time is the effective product of a phase transition, this interpretation may be dubious. 
colored fields can be expanded into Fourier modes

$$
\varphi_{1,2,3,4}^{a}=\sum_{p_{j} \in \mathbb{Z}} \varphi_{\left[p_{j}\right]}^{a} e^{i p_{1} \theta_{1}} e^{i p_{2} \theta_{2}} e^{i p_{3} \theta_{3}} e^{i p_{4} \theta_{4}}, \quad \theta_{i} \in[0,2 \pi), \quad\left[p_{j}\right]=\left(p_{1}, p_{2}, p_{3}, p_{4}\right) .
$$

where the group elements $h_{i} \in U(1)$. We adopt the notation $\varphi^{a}\left(h_{1}, h_{2}, h_{3}, h_{4}\right)=\varphi_{1,2,3,4}^{a}$. Remark that no symmetry under permutation of arguments is assumed for any of the field $\varphi^{a}$ and for the corresponding tensors $\varphi_{\left[p_{j}\right]}^{a}$.

The kinetic part of the action for the last four fields is the standard "local" colored one

$$
S^{\mathrm{kin}, 1,2,3,4}=\sum_{a=1}^{4} \int_{h_{j}} \bar{\varphi}_{1,2,3,4}^{a} \varphi_{1,2,3,4}^{a} .
$$

The symbol $\int_{h_{j}}$ stands for the Haar measure over all group variables with label of the form $h_{j}$. For each variable, this is merely the normalized compact integral $(1 / 2 \pi) \int_{0}^{2 \pi} d \theta_{j}$.

The interaction part of the action is the standard colored action in 4 dimensions [37]

$$
\begin{aligned}
S^{\mathrm{int}} & =\tilde{\lambda} \int_{h_{i j}} \varphi_{1,2,3,4}^{0} \varphi_{4,5,6,7}^{1} \varphi_{7,3,8,9}^{2} \varphi_{9,6,2,10}^{3} \varphi_{10,8,5,1}^{4} \\
& +\tilde{\tilde{\lambda}} \int_{h_{i j}}^{4} \bar{\varphi}_{1,2,3,4}^{0} \bar{\varphi}_{4,5,6,7}^{1} \bar{\varphi}_{7,3,8,9}^{2} \bar{\varphi}_{9,6,2,10}^{3} \bar{\varphi}_{10,8,5,1}^{4},
\end{aligned}
$$

where $\tilde{\lambda}$ and $\tilde{\bar{\lambda}}$ are coupling constants.

We want to build a model in which the field with color 0 is singled out and is the only dynamical field. Hence for that single field we introduce a different propagator with quadratic action

$$
S^{\mathrm{kin}, 0}=\int_{h_{j}} \bar{\varphi}_{1,2,3,4}^{0}\left(-\sum_{s=1}^{4} \Delta_{s}+m^{2}\right) \varphi_{1,2,3,4}^{0},
$$

where $\Delta_{s}:=\partial_{(s) \theta}^{2}$ denotes the Laplacian on $U(1) \equiv S^{1}$ acting on the strand index $s$. The corresponding Gaussian measure of covariance $C=\left(-\sum_{s} \Delta_{s}+m^{2}\right)^{-1}$ is noted as $d \mu_{C}$.

We integrate over the four colors 1,2,3,4 and obtain a partition function with an effective action for the last tensor $\varphi^{0}$ [49]:

$$
\begin{aligned}
& Z=\int d \mu_{C}\left[\varphi^{0}\right] e^{-S^{\mathrm{int}, 0}}, \\
& S^{\mathrm{int}, 0}=\sum_{\mathcal{B}} \frac{(\tilde{\lambda} \tilde{\tilde{\lambda}})_{\mathcal{B}}}{\operatorname{Sym}(\mathcal{B})} N^{f(p, D)-\frac{2}{(D-2) !} \omega(\mathcal{B})} \operatorname{Tr}_{\mathcal{B}}\left[\bar{\varphi}^{0} \varphi^{0}\right],
\end{aligned}
$$

where the sum in $\mathcal{B}$ is performed on all bubbles, or connected vacuum graphs with colors 1 up to $D$ and $p$ vertices; $f(p, D)$ is a positive function of the number of vertices and the dimension; $\omega(\mathcal{B}):=\sum_{J} g_{J}$ is the sum of genera of sub-ribbon graphs called jackets $J$ of the bubble, and $\operatorname{Tr}_{\mathcal{B}}\left[\bar{\varphi}^{0} \varphi^{0}\right]$ are called tensor network operators. Graphs with $\omega(\mathcal{B})=0$ are called melons. Non melonic contributions defined by $\omega(\mathcal{B})>0$ are clearly suppressed from (5). For 


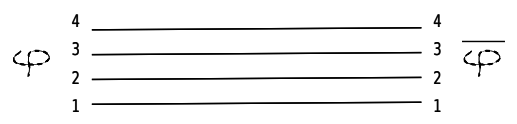

Figure 1: The propagator.
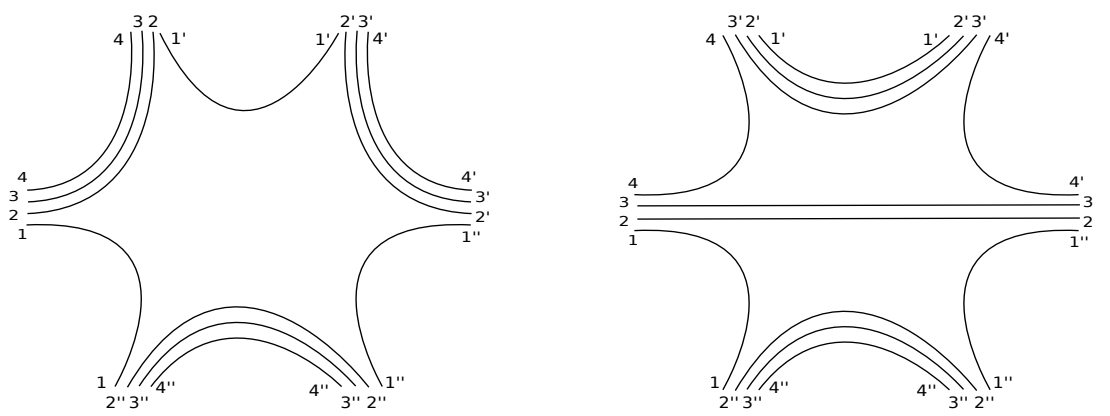

Figure 2: Vertices of the type $V_{6 ; 1}$ (left) and $V_{6 ; 2}$ (right).

details on all this terminology we refer to [44] and references therein. We will concentrate only on the melonic sector of the theory. A fundamental idea of [49] is to attribute a different coupling constant to different tensor network operators. We simply write (dropping from now on the last color index 0)

$$
S^{\text {int }, 0}=\sum_{\mathcal{B}} \frac{\lambda_{\mathcal{B}}}{\operatorname{Sym}(\mathcal{B})} \operatorname{Tr}_{\mathcal{B}}[\bar{\varphi} \varphi] .
$$

In order to get a renormalizable theory, we have to truncate this action to a finite number of marginal and relevant terms, in renormalization group language.

The trace operators or effective interaction terms that we will consider in the following are monomials of order six at most, given by

$$
\begin{aligned}
S_{6 ; 1}= & \int_{h_{j}} \varphi_{1,2,3,4} \bar{\varphi}_{1^{\prime}, 2,3,4} \varphi_{1^{\prime}, 2^{\prime}, 3^{\prime}, 4^{\prime}} \bar{\varphi}_{1^{\prime \prime}, 2^{\prime}, 3^{\prime}, 4^{\prime}} \varphi_{1^{\prime \prime}, 2^{\prime \prime}, 3^{\prime \prime}, 4^{\prime \prime}} \bar{\varphi}_{1,2^{\prime \prime}, 3^{\prime \prime}, 4^{\prime \prime}} \\
& + \text { permutations }, \\
S_{6 ; 2}= & \int_{h_{j}} \varphi_{1,2,3,4} \bar{\varphi}_{1^{\prime}, 2^{\prime}, 3^{\prime}, 4} \varphi_{1^{\prime}, 2^{\prime}, 3^{\prime}, 4^{\prime}} \bar{\varphi}_{1^{\prime \prime}, 2,3,4^{\prime}} \varphi_{1^{\prime \prime}, 2^{\prime \prime}, 3^{\prime \prime}, 4^{\prime \prime}} \bar{\varphi}_{1,2^{\prime \prime}, 3^{\prime \prime}, 4^{\prime \prime}} \\
& + \text { permutations }, \\
S_{4 ; 1}= & \int_{h_{j}} \varphi_{1,2,3,4} \bar{\varphi}_{1^{\prime}, 2,3,4} \varphi_{1^{\prime}, 2^{\prime}, 3^{\prime}, 4^{\prime}} \bar{\varphi}_{1,2^{\prime}, 3^{\prime}, 4^{\prime}}+\text { permutations },
\end{aligned}
$$

where the sum is over all 24 permutations of the four color indices.

Feynman graphs are tensor like: fields are represented by half lines with four strands, propagators are lines with the same structure (see Fig.1), meanwhile, vertices are non local objects as depicted in Fig 2 and 3. For convenience, we will sometimes use simplified diagrammatics where the strand structure will be hidden.

Furthermore, the renormalization analysis of Section 6 also leads to add to the action 

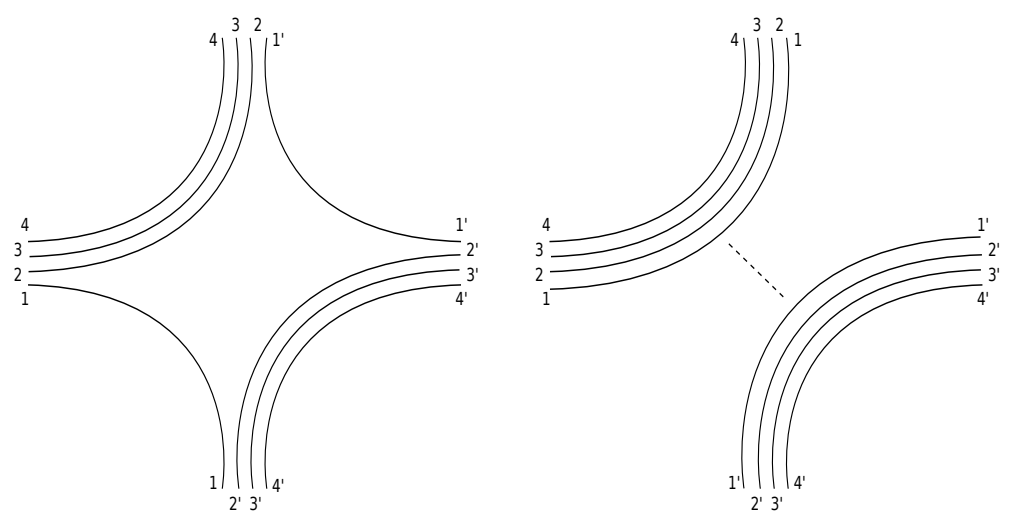

Figure 3: Vertices of the type $V_{4 ; 1}$ (left) and $V_{4 ; 2}$ (right).

another $\varphi^{4}$-type anomalous divergent term, namely:

$$
S_{4 ; 2}=\left[\int_{h_{j}} \bar{\varphi}_{1,2,3,4} \varphi_{1,2,3,4}\right]\left[\int_{h_{j}^{\prime}} \bar{\varphi}_{1^{\prime}, 2^{\prime}, 3^{\prime}, 4^{\prime}} \varphi_{1^{\prime}, 2^{\prime}, 3^{\prime}, 4^{\prime}}\right] .
$$

The interaction (10) can be considered as a joined pair of two factorized $\varphi^{2}$ vertices which we represent as two lines with a dotted line between them, see Fig. 3 .

In the next section we shall introduce an ultraviolet cutoff $\Lambda$ on the propagator, which becomes $C^{\Lambda}$. We introduce as usual bare and renormalized couplings, the difference of which are coupling constants counterterms, called $C T$. We have also to introduce counterterms in the bare action to perform the mass and wave function renormalization hence we also define quadratic terms in the action:

$$
S_{2 ; 1}=\int_{h_{j}} \bar{\varphi}_{1,2,3,4} \varphi_{1,2,3,4}, \quad S_{2 ; 2}=\int_{h_{j}} \bar{\varphi}_{1,2,3,4}\left(-\sum_{s=1}^{4} \Delta_{s}\right) \varphi_{1,2,3,4} .
$$

The propagator $C$ has for coefficients the renormalized mass $m^{2}$ and the renormalized wave function 1.

The action of the model is then defined as

$$
S^{\Lambda}=\lambda_{6 ; 1}^{\Lambda} S_{6 ; 1}+\lambda_{6 ; 2}^{\Lambda} S_{6 ; 2}+\lambda_{4 ; 1}^{\Lambda} S_{4 ; 1}+\lambda_{4 ; 2}^{\Lambda} S_{4 ; 2}+C T_{2 ; 1}^{\Lambda} S_{2 ; 1}+C T_{2 ; 2}^{\Lambda} S_{2 ; 2}
$$

and the partition function is

$$
Z=\int d \mu_{C^{\Lambda}}[\varphi] e^{-S^{\Lambda}}
$$

The renormalization theorem means that we can define four renormalized coupling constants $\lambda_{6 ; 1}^{\text {ren }}, \lambda_{6 ; 2}^{\text {ren }}, \lambda_{4 ; 1}^{\text {ren }}, \lambda_{4 ; 2}^{\text {ren }}$ such that choosing appropriately the 6 counterterms power series then the power series expansion of any Schwinger function of the model expressed in powers of the renormalized couplings has a finite limit at all orders. More precisely 
Theorem 1. There exist 6 counterterms $C T_{6 ; 1}^{\Lambda}, C T_{6 ; 2}^{\Lambda}, C T_{4 ; 1}^{\Lambda}, C T_{4 ; 2}^{\Lambda}, C T_{2 ; 1}^{\Lambda}, C T_{2 ; 2}^{\Lambda}$, each of which a multi-power series of the four renormalized couplings $\left(\lambda_{6 ; 1}^{r e n}, \lambda_{6 ; 2}^{r e n}, \lambda_{4 ; 1}^{\text {ren }}, \lambda_{4 ; 2}^{\text {ren }}\right)$, with $\Lambda$ dependent coefficients, such that $C T_{6 ; 1}^{\Lambda}, C T_{6 ; 2}^{\Lambda}, C T_{4 ; 1}^{\Lambda}, C T_{4 ; 2}^{\Lambda}$ have valuation at least 2 and $C T_{2 ; 1}^{\Lambda}, C T_{2 ; 2}^{\Lambda}$ have valuation at least 1 , and such that if the bare couplings in equation are defined as

$$
\begin{aligned}
& \lambda_{6 ; 1}^{\Lambda}=\lambda_{6 ; 1}^{r e n}+C T_{6 ; 1}^{\Lambda}\left(\lambda_{6 ; 1}^{r e n}, \lambda_{6 ; 2}^{r e n}, \lambda_{4 ; 1}^{r e n}, \lambda_{4 ; 2}^{r e n}\right) \\
& \lambda_{6 ; 2}^{\Lambda}=\lambda_{6 ; 2}^{r e n}+C T_{6 ; 2}^{\Lambda}\left(\lambda_{6 ; 1}^{r e n}, \lambda_{6 ; 2}^{r e n}, \lambda_{4 ; 1}^{r e n}, \lambda_{4 ; 2}^{r e n}\right) \\
& \lambda_{4 ; 1}^{\Lambda}=\lambda_{4 ; 1}^{r e n}+C T_{4 ; 1}^{\Lambda}\left(\lambda_{6 ; 1}^{r e n}, \lambda_{6 ; 2}^{r e n}, \lambda_{4 ; 1}^{r e n}, \lambda_{4 ; 2}^{r e n}\right) \\
& \lambda_{4 ; 2}^{\Lambda}=\lambda_{4 ; 2}^{r e n}+C T_{4 ; 2}^{\Lambda}\left(\lambda_{6 ; 1}^{r e n}, \lambda_{6 ; 2}^{r e n}, \lambda_{4 ; 1}^{r e n}, \lambda_{4 ; 2}^{r e n}\right)
\end{aligned}
$$

then the Schwinger functions of the model with partition function 13, when re-expressed as multi-power series in the four renormalized couplings $\left(\lambda_{6 ; 1}^{r e n}, \lambda_{6 ; 2}^{\text {ren }}, \lambda_{4 ; 1}^{\text {ren }}, \lambda_{4 ; 2}^{r e n}\right)$, have all their coefficients finite when the ultraviolet cutoff $\Lambda$ goes to infinity.

This is the usual statement of perturbative renormalizability of the model. The rest of the paper is devoted to the proof of this theorem.

\section{Multiscale Analysis}

In this section, we define the multiscale analysis leading to the power counting and proof of Theorem 1. First, we need a bound on the propagator adapted to the scale analysis. Then, we apply the usual multiscale formalism [55]. We obtain a prime power counting of the amplitude of any graph in term of its high or quasi-local subgraphs. The fine analysis of this power counting and renormalization program will be differed to the next sections.

\subsection{Decomposition and bounds on the propagator}

Let us consider the $U(1)$ tensor dynamical model defined by the kinetic term

$$
\widehat{S}^{\text {kin }}=\int_{h_{j}} \bar{\varphi}_{1,2,3,4}\left(-\sum_{s=1}^{4} \Delta_{s}+m^{2}\right) \varphi_{1,2,3,4} .
$$

Given a set of integers $\left(\left\{q_{s}\right\} ;\left\{q_{s}^{\prime}\right\}\right):=\left(\left\{q_{1}, q_{2}, q_{3}, q_{4}\right\} ;\left\{q_{1}^{\prime}, q_{2}^{\prime}, q_{3}^{\prime}, q_{4}^{\prime}\right\}\right), q_{s}, q_{s}^{\prime} \in \mathbb{Z}$, the kernel of the propagator $\left[-\sum_{s=1}^{4} \Delta_{s}+m^{2}\right]^{-1}$ in momentum space can be written

$$
C\left(\left\{q_{s}\right\} ;\left\{q_{s}^{\prime}\right\}\right)=\left[\sum_{s=1}^{4}\left(q_{s}\right)^{2}+m^{2}\right]^{-1}\left[\prod_{s=1}^{4} \delta_{q_{s}, q_{s}^{\prime}}\right] .
$$

Choosing a local coordinate system on $S^{1} \sim U(1)$, parameterized by $\theta \in[0,2 \pi)$, consider a set of such coordinates $\left(\left\{\theta_{s}\right\} ;\left\{\theta_{s}^{\prime}\right\}\right):=\left(\left\{\theta_{1}, \theta_{2}, \theta_{3}, \theta_{4}\right\} ;\left\{\theta_{1}^{\prime}, \theta_{2}^{\prime}, \theta_{3}^{\prime}, \theta_{4}^{\prime}\right\}\right)$ and the corresponding elements $\left(\left\{h_{s}\right\} ;\left\{h_{s}^{\prime}\right\}\right)$ such that $h_{s}=e^{i q_{s} \theta_{s}}, s=1, \ldots, 4$. The kernel (19) expressed in the direct space can be evaluated as

$$
C\left(\left\{\theta_{s}\right\} ;\left\{\theta_{s}^{\prime}\right\}\right)=\sum_{q_{s}, q_{s}^{\prime} \in \mathbb{Z}} C\left(\left\{q_{s}\right\} ;\left\{q_{s}^{\prime}\right\}\right) e^{i \sum_{s}\left[q_{s} \theta_{s}-q_{s}^{\prime} \theta_{s}^{\prime}\right]}=\sum_{q_{s} \in \mathbb{Z}} \int_{0}^{\infty} e^{-\alpha\left[\sum_{s} q_{s}^{2}+m^{2}\right]+i \sum_{s} q_{s}\left(\theta_{s}-\theta_{s}^{\prime}\right)} d \alpha,
$$


where we have introduced a Schwinger parameter $\alpha$.

A direct calculation yields, up to some unessential constant $k=\pi^{2}$

$$
\begin{aligned}
C\left(\left\{\theta_{s}\right\} ;\left\{\theta_{s}^{\prime}\right\}\right) & =k \int_{0}^{\infty} \frac{e^{-m^{2} \alpha}}{\alpha^{2}} e^{-\frac{1}{4 \alpha} \sum_{s}\left[\theta_{s}-\theta_{s}^{\prime}\right]^{2}} T\left(\alpha ;\left\{\theta_{s}\right\} ;\left\{\theta_{s}^{\prime}\right\}\right) d \alpha, \\
T\left(\alpha ;\left\{\theta_{s}\right\} ;\left\{\theta_{s}^{\prime}\right\}\right) & =\prod_{s=1}^{4}\left\{1+2 \sum_{n=1}^{\infty} e^{-\frac{\pi^{2} n^{2}}{\alpha}} \cosh \left[\frac{n \pi}{\alpha}\left[\theta_{s}-\theta_{s}^{\prime}\right]\right]\right\},
\end{aligned}
$$

where $T$ can be related to the third Jacobi elliptic function (although this special function is not used in this paper) [56]. This is the general expression of the covariance in this $U(1)$ theory, which is the simplest finite volume four-dimensional theory with periodic boundary conditions on the Laplacian. The latter is an important feature of this theory: amplitudes and functions involving the quantities $\left|\theta_{s}-\theta_{s}^{\prime}\right|$ will be all translation invariant as functions on the torus.

In the following developments, we do not actually need the explicit expression of this propagator but only its behavior at small distance will be useful. The problem of infrared divergences is simply avoided in this paper by the fact that $U(1)$ is compact and for simplicity we can even assume $m^{2}=0$, to have no problem with the zero mode of the propagator.

We introduce the usual slice decomposition of the propagator:

$$
\begin{aligned}
& C=\sum_{i=0}^{\infty} C_{i}, \\
& C_{0}\left(\left\{\theta_{s}\right\} ;\left\{\theta_{s}^{\prime}\right\}\right)=k \int_{1}^{\infty} \frac{e^{-m^{2} \alpha}}{\alpha^{2}} e^{-\frac{1}{4 \alpha} \sum_{s}\left[\theta_{s}-\theta_{s}^{\prime}\right]^{2}} T\left(\alpha ;\left\{\theta_{s}\right\} ;\left\{\theta_{s}^{\prime}\right\}\right) d \alpha, \\
& C_{i}\left(\left\{\theta_{s}\right\} ;\left\{\theta_{s}^{\prime}\right\}\right)=k \int_{M^{-2(i+1)}}^{M^{-2 i}} \frac{e^{-m^{2} \alpha}}{\alpha^{2}} e^{-\frac{1}{4 \alpha} \sum_{s}\left[\theta_{s}-\theta_{s}^{\prime}\right]^{2}} T\left(\alpha ;\left\{\theta_{s}\right\} ;\left\{\theta_{s}^{\prime}\right\}\right) d \alpha .
\end{aligned}
$$

The following statement holds

Lemma 1. For all $i=0,1, \ldots$, for all $\underline{m} \in \mathbb{N}$ there exist some constants $K \geq 0, K_{\underline{m}} \geq 0$ and $\delta \geq 0$ such that

$$
\begin{aligned}
& C_{i}\left(\left\{\theta_{s}\right\} ;\left\{\theta_{s}^{\prime}\right\}\right) \leq K M^{2 i} e^{-\delta M^{i} \sum_{s=1}^{4}\left|\theta_{s}-\theta_{s}^{\prime}\right|}, \\
& \left(\prod_{k=1}^{\underline{m}} \partial_{\theta^{\prime}, s_{k}}\right) C_{i}\left(\left\{\theta_{s}\right\} ;\left\{\theta_{s}^{\prime}\right\}\right) \leq K_{\underline{m}} M^{(2+\underline{m}) i} e^{-\delta M^{i} \sum_{s=1}^{4}\left|\theta_{s}-\theta_{s}^{\prime}\right|} .
\end{aligned}
$$

Proof. For small $\alpha$, the propagator can be faithfully approximated by a heat kernel. In a high slice $i \gg 1$, the following bound is valid (see Appendix A for the calculation details)

$$
C_{i}\left(\left\{\theta_{s}\right\} ;\left\{\theta_{s}^{\prime}\right\}\right) \leq K^{\prime} \int_{M^{-2(i+1)}}^{M^{-2 i}} d \alpha \frac{e^{-m^{2} \alpha}}{\alpha^{2}} e^{-\frac{1}{4 \alpha} \sum_{s}\left[\theta_{s}-\theta_{s}^{\prime}\right]^{2}} \leq K^{\prime \prime} M^{2 i} e^{-\delta^{\prime} M^{2 i} \sum_{s}\left|\theta_{s}-\theta_{s}^{\prime}\right|^{2}}
$$

where $K^{\prime}, K^{\prime \prime}$ and $\delta^{\prime}$ are constants. From this last expression, we get the useful bound

$$
C_{i}\left(\left\{\theta_{s}\right\} ;\left\{\theta_{s}^{\prime}\right\}\right) \leq K M^{2 i} e^{-\delta M^{i} \sum_{s}\left|\theta_{s}-\theta_{s}^{\prime}\right|}
$$


with $K$ and $\delta$ some constants and the sum is performed over $s=1, \ldots, 4$. For the last slice, we have (see Appendix A

$$
C_{0}\left(\left\{\theta_{s}\right\} ;\left\{\theta_{s}^{\prime}\right\}\right) \leq K^{\prime} \int_{1}^{\infty} \frac{e^{-m^{2} \alpha / 2}}{\alpha^{2}} d \alpha \leq K e^{-\delta \sum_{s}\left|\theta_{s}-\theta_{s}^{\prime}\right|},
$$

where we used the fact that $\left|\theta_{s}-\theta_{s}^{\prime}\right| \leq 2 \pi$. This proves the first bound (23).

For the second inequality, we can differentiate $\underline{m}$ times the propagator with respect to a set of strands and get (note that we reintroduce the momentum space representation as intermediate step using a slice decomposition from (20) for simplifications)

$$
\begin{aligned}
& \left|\left(\prod_{k=1}^{\underline{m}} \partial_{\theta^{\prime}, s_{k}}\right) C_{i}\left(\left\{\theta_{s}\right\} ;\left\{\theta_{s}^{\prime}\right\}\right)\right| \leq \\
& \left|\sum_{q_{s} \in \mathbb{Z}} \int_{M^{-2(i+1)}}^{M^{-2 i}} \frac{1}{\alpha^{\underline{m} / 2}}\left[\prod_{k=1}^{\underline{m}}\left(-i \sqrt{\alpha} q_{s_{k}^{\prime}}\right)\right] e^{-\alpha\left[\sum_{s} q_{s}^{2}+m^{2}\right]} e^{i \sum_{s} q_{s}\left[\theta_{s}-\theta_{s}^{\prime}\right]} d \alpha\right| \\
& \leq K_{\underline{m}}^{\prime}\left|\sum_{q_{s} \in \mathbb{Z}} \int_{M^{-2(i+1)}}^{M^{-2 i}} \frac{1}{\alpha^{\underline{m} / 2}} e^{-\alpha\left[\sum_{s} q_{s}^{2}+m^{2}\right]} e^{i \sum_{s} q_{s}\left[\theta_{s}-\theta_{s}^{\prime}\right]} d \alpha\right| \leq K_{\underline{m}} M^{2 i+2(\underline{m} / 2) i} e^{-\delta M^{i} \sum_{s}\left|\theta_{s}-\theta_{s}^{\prime}\right|},
\end{aligned}
$$

where, in the last stage, we perform the summation in $q$ 's and use again the bound on $T$ (according to the same procedure yielding (25)).

Similarly, for the last slice, we have

$$
\left|\left(\prod_{k=1}^{\underline{m}} \partial_{\theta^{\prime}, s_{k}}\right) C_{0}\left(\left\{\theta_{s}\right\} ;\left\{\theta_{s}^{\prime}\right\}\right)\right| \leq K^{\prime} \int_{1}^{\infty} \frac{e^{-m^{2} \alpha / 2}}{\alpha^{2+\underline{m} / 2}} d \alpha \leq K_{m} e^{-\delta \sum_{s}\left|\theta_{s}-\theta_{s}^{\prime}\right|} .
$$

Imposing an ultraviolet cutoff consists in summing the slice index only up to a large integer $\Lambda$ in 22

$$
C^{\Lambda}=\sum_{i=0}^{\Lambda} C_{i}
$$

and the ultraviolet limit is $\Lambda \rightarrow \infty$. From now on, we forget the superscript $\Lambda$ most of the time for simplicity.

\subsection{Momentum attributions and optimal amplitude bound}

Let us consider a connected amputated graph $\mathcal{G}$ with set of vertices $\mathcal{V}$, with cardinal $V=|\mathcal{V}|$, and $\mathcal{L}$ set of lines, with cardinal $L=|\mathcal{L}|$. Let $N_{\text {ext }}$ be the number of external fields or legs. Since wave-function counterterms have special power counting because they carry an extra $p^{2}$, we suppose first for simplicity that the graph does not have wave-function counterterm, then we add the easy correction for wave function counterterms.

Direct space - The bare amplitude associated with $\mathcal{G}$ is of the form

$$
A_{\mathcal{G}}=\sum_{\mu} \int\left[\prod_{v, s} d \theta_{v, s}\right]\left[\prod_{\ell \in \mathcal{L}} C_{i_{\ell}(\mu)}\left(\left\{\theta_{v, \ell(v), s}\right\} ;\left\{\theta_{v^{\prime}, \ell\left(v^{\prime}\right), s}\right\}\right)\right]\left[\prod_{v \in \mathcal{V} ; s} \delta\left(\theta_{v, s}-\theta_{v, s^{\prime}}\right)\right]
$$


where $\theta_{v, \ell(v), s}$ are coordinates involved in the propagator which should possess a vertex label $v$, a strand label $s$ but also a line index $i_{\ell} ; \theta_{v s}$ are the same position coordinates involved in the vertex which should have both vertex $v$ and strand $s$ labels; $\delta\left(\theta_{v, s}-\theta_{v, s^{\prime}}\right)$ is the delta Dirac distribution on the torus; $\mu=\left(i_{1}, i_{2}, \ldots, i_{q}\right)$ is a multi-index called momentum assignment which gives to each propagator of each internal line $\ell$ of the graph a scale $i_{\ell} \in[0, \Lambda]$; the sum over $\mu$ is performed on all possible assignments. The graph being amputated, there is no external propagator but rather external vertices where test functions or external fields can be hooked. It is conventional to give a fixed scale $i_{\text {ext }}=-1$ for those external lines. We focus on $A_{\mathcal{G} ; \mu}$. The sum $A_{\mathcal{G}}=\sum_{\mu} A_{\mathcal{G} ; \mu}$ can be done only after renormalization.

The next stage is to perform some spatial integrations of the $\theta_{v, s}$ vertex variables in $A_{\mathcal{G} ; \mu}$. The main point is to bound this integral in an "optimal" way.

Given a momentum assignment $\mu$ and a fixed scale $i$, we consider the complete list of the connected components $G_{i}^{(k)}, k=1,2, \ldots, k(i)$, of the subgraph $\mathcal{G}_{i}$ made of all lines in $\mathcal{G}$ with the scale attribution $j \geq i$ in $\mu$. These subgraphs called high or quasi-local are the key objects in the multiscale expansion [55]. A partial (inclusion) order can be defined on the set of $G_{i}^{(k)}$ and $\mathcal{G}_{0}=\mathcal{G}$. The abstract tree made of nodes as the $G_{i}^{(k)}$ associated to that partial order is called the Gallavotti-Nicolò tree [57], for which $\mathcal{G}$ is merely the root. Given an arbitrary subgraph $g$, one defines:

$$
i_{g}(\mu)=\inf _{l \in g} i_{l}(\mu), \quad e_{g}(\mu)=\sup _{l \text { external line of } g} i_{l}(\mu) .
$$

The first quantity is the lowest scale inside the subgraph $g$ whereas the second corresponds to the higher scale of all lines (external to $g$ ) to which the subgraph $g$ is hooked. The subgraph $g$ is a $G_{i}^{(k)}$ for a given $\mu$ if and only if $i_{g}(\mu) \geq i>e_{g}(\mu)$, in other words, any internal scale is higher than the greater external scale. In the ordinary field theory situation, the key point is to optimize the bound over spatial integrations by choosing a spanning tree $T$ of $\mathcal{G}$ 6 compatible with the abstract Gallavotti-Nicolò tree. This can be done by considering the restriction $T_{i}^{k}$ of $T$ to any $G_{i}^{(k)}$ in such way that $T_{i}^{k}$ is still a spanning tree for $G_{i}^{(k)}$.

The present situation is slightly different. Due to the particular form of the vertex operator, i.e. a product of delta functions, the graph amplitude $A_{\mathcal{G}}$ factorizes in term of closed or open circuit called "faces". This notion coincides with ribbon graph faces in matrix model, see for instance [58]. Let $\mathcal{F}$ be the set of such faces which can be decomposed in closed or internal faces, say $\mathcal{F}_{\text {int }}$, and open faces which touch on external vertices, we call them $\mathcal{F}_{\text {ext }}$. The cardinal $F$ of $\mathcal{F}$ is of course the sum of $F_{\text {ext }}$ and $F_{\text {int }}$ cardinal of $\mathcal{F}_{\text {int }}$ and $\mathcal{F}_{\text {ext }}$, respectively.

We write, using at first the bound (23) (and dropping some indices, for simplicity $i_{\ell}(\mu)=$ $\left.i_{\ell}\right)$,

$$
\begin{aligned}
\left|A_{\mathcal{G} ; \mu}\right| & \leq \int\left[\prod_{v} d \theta_{v, s}\right]\left[\prod_{\ell \in \mathcal{L}} K M^{2 i_{\ell}} e^{-\delta M^{i_{\ell}} \sum_{s=1}^{4}\left|\theta_{v, i_{\ell}, s}-\theta_{v, i_{\ell}, s}^{\prime}\right|}\right] \prod_{v \in \mathcal{V}} \delta\left(\theta_{v, s}-\theta_{v, s^{\prime}}\right) \\
& \leq\left[\prod_{\ell \in \mathcal{L}} K M^{2 i_{\ell}}\right] \int\left[\prod_{f \in \mathcal{F}} \prod_{\ell \in f} d \theta_{\ell, f}\right] \prod_{f \in \mathcal{F}} \prod_{\ell \in f} e^{-\delta M^{i_{\ell}}\left|\theta_{\ell, f}-\theta_{\ell, f}^{\prime}\right|} .
\end{aligned}
$$

\footnotetext{
${ }^{6}$ A spanning tree of $\mathcal{G}$ is a set of lines passing through all vertices of $\mathcal{G}$ without forming loop. Integrations on vertex variables will be associated with the choice of $T$.
} 
In the last step, we simply rewrite the amplitude in terms of faces and the product $\prod_{\ell \in f}$ is performed over all lines (here strands) from which a given face $f$ is built. After this factorization, each variable $\theta$ can be now indexed by a couple $(\ell, f)$, i.e. by a unique face and a line where it can appear. Each variable can only appear (at most) in two such lines.

Each integration in position coordinates $\theta$ will bring a "good" factor of $M^{-i}$. We therefore need to integrate as much as possible position coordinates with decay factors from high indices $i_{\ell}$ in order to bring more convergence. There is a way to optimize the bound on position integrations but, first, let us define the "scale of a strand" as the same scale of the line generating this strand. We can integrate all positions but one in each open or closed faces. Along any open face of a $G_{i}^{(k)}$, we can integrate all positions but one; each integration will give a factor $M^{-i_{\ell}}$ corresponding to the strand (and its scale index) where the integrated variable belongs. It remains a last integration with respect to a variable touching an external strand. It will be made later with a lower line and that will bring a larger factor of $M^{-j}$, with $j \leq i_{\ell}-1$. Hence for an open face, all internal decays can be used once. For closed faces, the problem is similar but one last integration cannot be performed with scaled decays. The integration of the closed face can be optimized by simply choosing the highest scales of strands belonging to the face. This integration, similar to a momentum routine, will be performed on position labels on a tree $T_{f}$ (this tree is not a tree of lines as in ordinary quantum field theory, but a tree made of strands which possess also a scale index) associated with the face $f$. If $f$ is open $T_{f}=f$, if $f$ is closed $T_{f} \subsetneq f$ and $T_{f}$ consists in the set of all strands of $f$ save one. The tree $T_{f} \subseteq f$ (which is the analog of the spanning tree $T$ ) will be chosen to be compatible with the abstract Gallavoti-Nicolò tree associated with the $G_{i}^{(k)}$ in the sense that, the restriction $T_{f, i}^{k}=T_{f} \cap G_{i}^{(k)}$ is an open face in $G_{i}^{(k)}$ (see Fig. 4). The set of $T_{f, i}^{k}$ is called a "spanning forest" and is made of a set of connected strands, a tree, belonging to the same face.

We rewrite each factor in terms of $G_{i}^{(k)}$ in the manner of [55]:

$$
\begin{aligned}
& \prod_{\ell \in \mathcal{G}} M^{2 i_{\ell}}=\prod_{\ell \in \mathcal{G}} \prod_{i=1}^{i_{\ell}} M^{2}=\prod_{\ell \in \mathcal{G}} \prod_{(i, k) \in \mathbb{N}^{2} / \ell \in G_{i}^{(k)}} M^{2}=\prod_{(i, k) \in \mathbb{N}^{2}} \prod_{\ell \in G_{i}^{(k)}} M^{2}=\prod_{(i, k) \in \mathbb{N}^{2}} M^{2 L\left(G_{i}^{(k)}\right)} ; \\
& {\left[\prod_{f \in \mathcal{F}_{\text {ext }}} \prod_{\ell \in f} M^{-i_{\ell}}\right]\left[\prod_{f \in \mathcal{F}_{\text {int }}} \prod_{\ell \in T_{f} \subset f} M^{-i_{\ell}}\right]=\left[\prod_{f \in \mathcal{F}_{\text {ext }}} \prod_{\ell \in f} \prod_{i=1}^{i_{\ell}} M^{-1}\right]\left[\prod_{f \in \mathcal{F}_{\text {int }}} \prod_{\ell \in T_{f} \subset f} \prod_{i=1}^{i_{\ell}} M^{-1}\right]} \\
& =\left[\prod_{f \in \mathcal{F}_{\text {ext }}} \prod_{\ell \in f} \prod_{(i, k) \in \mathbb{N}^{2} / \ell \in G_{i}^{(k)}} M^{-1}\right]\left[\prod_{f \in \mathcal{F}_{\text {int }}} \prod_{\ell \in T_{f} \subset f} \prod_{(i, k) \in \mathbb{N}^{2} / \ell \in G_{i}^{(k)}} M^{-1}\right] \\
& =\prod_{(i, k)}\left[\left[\prod_{f \in \mathcal{F}_{\text {ext }} \cap G_{i}^{(k)}} \prod_{\ell \in T_{f, i}^{k}=f \cap G_{i}^{(k)}} M^{-1}\right]\left[\prod_{f \in \mathcal{F}_{\text {int }} \cap G_{i}^{(k)}} \prod_{\ell \in T_{f, i}^{k}=T_{f} \cap G_{i}^{(k)}} M^{-1}\right]\right] \\
& =\prod_{(i, k)} \prod_{f \in \mathcal{F} \cap G_{i}^{(k)}} \prod_{\ell \in T_{f, i}^{k}} M^{-1}=\prod_{(i, k)} M^{-4 L\left(G_{i}^{(k)}\right)+F_{\text {int }}\left(G_{i}^{(k)}\right)},
\end{aligned}
$$

where $L\left(G_{i}^{(k)}\right)$ and $F_{\text {int }}\left(G_{i}^{(k)}\right)$ denote the number of internal lines and internal faces of the subgraph $G_{i}^{(k)}$, respectively. In the last equality, we use the fact that, given line at scale $i$, all 4 strand positions can be integrated and then they should contribute to the same the subgraph $G_{i}^{(k)}$. However, since one position label per closed face is not integrated (with any 


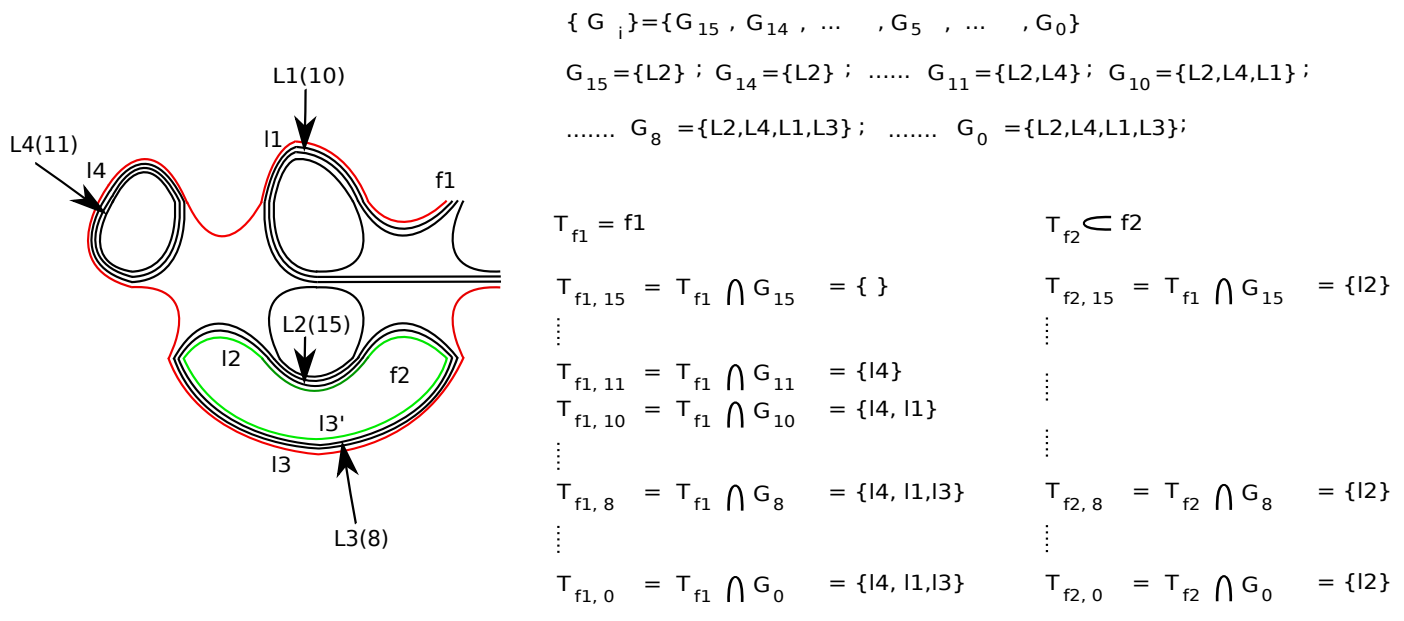

Figure 4: A graph with a given scale attribution: lines of the graph are $\{L 1, L 2, L 3, L 4\}$ with scale $\{10,15,8,11\}$; the face $f 1$ (in red) is open and formed by the strands $l 1, l 4, l 3$; the face $f 2$ (in green) is closed and formed by $l 2, l 3^{\prime}$. All $G_{i}^{(k=1)}$ have a unique connected component. The trees $T_{f 1}$ and $T_{f 2}$ decomposed on scales appear as $T_{f 1, i}$ and $T_{f 2, i}$ the set of which forms a spanning forest of $f 1$ and $f 2$, respectively.

line decay) but is simply integrated over the torus (without decay), it brings a full factor 1. Therefore we have the relation

$$
4 L\left(G_{i}^{(k)}\right)=\sum_{f \in \mathcal{F}_{\text {ext }} \cap G_{i}^{(k)}}\left|T_{f, i}^{k}\right|+\sum_{f \in \mathcal{F}_{\text {int }} \cap G_{i}^{(k)}}\left(\left|T_{f, i}^{k}\right|+1\right), \quad F_{\text {int }}\left(G_{i}^{(k)}\right)=\sum_{f \in \mathcal{F}_{\text {int }} \cap G_{i}^{(k)}} 1 .
$$

Combining the results (34) with the factors coming from spatial integrations, we obtain a bound of the graph amplitude at a given attribution $\mu$

$$
\left|A_{\mathcal{G} ; \mu}\right| \leq K^{n} \prod_{(i, k)} M^{-2 L\left(G_{i}^{(k)}\right)+F_{\text {int }}\left(G_{i}^{(k)}\right)}
$$

where $K$ is some constant and $n$ is the number of vertices of the graph (assumed without wave-function counterterms).

Momentum basis - We briefly sketch in this paragraph how the same prime power counting can be recovered in the momentum basis. The action will mainly remain the same: each field has to be replaced by a tensor $\varphi_{m_{1}, m_{2}, m_{3}, m_{4}}$ and the interaction pattern will be the same but with respect to the discrete indices $m_{i} \in \mathbb{Z}$. In this representation, the propagator kernel is of the form (19) and we can use its parametric form. Decomposing the propagator in the same slices $C=\sum_{i} C_{i}$, we have

$$
C_{i}\left(\left\{q_{s}\right\} ;\left\{q_{s}^{\prime}\right\}\right)=\int_{M^{-2(i+1)}}^{M^{-2 i}} d \alpha e^{-\alpha\left[\left(q_{1}\right)^{2}+\left(q_{2}\right)^{2}+\left(q_{3}\right)^{2}+\left(q_{4}\right)^{2}+m^{2}\right]} \prod_{s=1}^{4} \delta_{q_{s}, q_{s}^{\prime}}
$$




$$
\begin{aligned}
\leq K M^{-2 i} e^{-\delta M^{-i}\left[\sum_{s}\left|q_{s}\right|+m^{2}\right]} \prod_{s=1}^{4} \delta_{q_{s}, q_{s}^{\prime}} \\
C_{1}\left(\left\{q_{s}\right\} ;\left\{q_{s}^{\prime}\right\}\right)=\int_{1}^{\infty} d \alpha e^{-\alpha\left[\left(q_{1}\right)^{2}+\left(q_{2}\right)^{2}+\left(q_{3}\right)^{2}+\left(q_{4}\right)^{2}+m^{2}\right]} \prod_{s=1}^{4} \delta_{q_{s}, q_{s}^{\prime}} \leq K \prod_{s=1}^{4} \delta_{q_{s}, q_{s}^{\prime}} .
\end{aligned}
$$

Given a momentum assignment $\mu$, the multiscale representation of a given amputated graph amplitude can be expressed as

$$
A_{\mathcal{G} ; \mu}=\sum_{q_{v, s}} \prod_{\ell \in \mathcal{L}} C_{i_{\ell}(\mu)}\left(\left\{q_{v, \ell(v), s}\right\} ;\left\{q_{v^{\prime}, \ell\left(v^{\prime}\right), s}\right\}\right) \prod_{v \in \mathcal{V} ; s} \delta_{q_{v, s}, q_{v, s^{\prime}}},
$$

the last $\delta$ 's are Kronecker symbols associated to vertices. The sum is performed on all integers $q_{v, s} \in \mathbb{Z}$ in the momentum basis. Using the fact that faces factor in the amplitude, we obtain

$$
\begin{aligned}
\left|A_{\mathcal{G} ; \mu}\right| & \leq K^{n} \prod_{\ell \in \mathcal{L}} M^{-2 i_{\ell}} \sum_{q_{s}} \prod_{\ell \in \mathcal{L}} \prod_{s=1}^{4} \delta_{q_{i_{\ell} s}, q_{\ell_{\ell}}^{\prime}} e^{-\delta M^{-i_{\ell}}\left[\sum_{s}\left|q_{s}\right|+m^{2}\right]} \\
& \leq K^{n} \prod_{\ell \in \mathcal{L}} M^{-2 i_{\ell}} \sum_{q_{f}} \prod_{f \in \mathcal{F}} \prod_{\ell \in f} e^{-\delta M^{-i_{\ell}}\left|q_{f}\right|},
\end{aligned}
$$

where the bound (37) has been used. We introduced also $q_{f}$ as momenta per face amplitude and the notation " $l \in f$ " to mention the particular line (in fact, strand) contributing to the face $f$. Two cases may occur: (1) the face $f \in \mathcal{F}_{\text {int }}$, then the face amplitude is of the form $\sum_{q_{f}} e^{-\sum_{\ell \in f} \delta M^{-i} \ell\left|q_{f}\right|}$. Presently, we optimize by taking the lowest possible $i_{\ell}$ in the face because, up to some constants $\delta, \delta^{\prime}, \sum_{p \in \mathbb{N}} e^{-\delta M^{-i} p}=\delta^{\prime} M^{i}+O\left(M^{-i}\right)$; (2) the face $f$ is open, then all sums in $q_{s}$ can be performed and one gets $O(1)$. Hence, the first step is to bound again the above amplitude by only terms involving $\mathcal{F}_{\text {int }}$. Its remains to choose a tree $T_{f}$ of each internal face $f \in \mathcal{F}_{\text {int }}$ compatible with the Gallavoti-Nicolò tree in the same way as done above. The contributions can be again recast in terms of the $G_{i}^{(k)}$ (once again by introducing the restriction of $\left.T_{f, i}^{k}=T_{f} \cap G_{i}^{(k)}\right)$. One infers the same bound as given by (36).

The previous bounds applies to connected graphs without wave function counterterm. If the graph contains such counterterm vertices of the type $S_{2 ; 2}$, let $V_{2}^{\prime}$ be their number. For each such counterterm, we have an extra $p^{2}$ hence $M^{2 i}$ factor. Hence, we finally have:

Lemma 2. For a connected graph $\mathcal{G}$ (with external arguments integrated versus fixed smooth test functions), we have

$$
\left|A_{\mathcal{G} ; \mu}\right| \leq K^{n} \prod_{(i, k)} M^{\omega_{d}\left(G_{i}^{(k)}\right)},
$$

where $K$ and $n$ are large constants, $\omega_{d}\left(G_{i}^{(k)}\right)=-2 L\left(G_{i}^{(k)}\right)+F_{\text {int }}\left(G_{i}^{(k)}\right)+2 V_{2}^{\prime}\left(G_{i}^{(k)}\right)$.

We call degree of divergence of the graph $\mathcal{G}$, the quantity

$$
\omega_{d}(\mathcal{G})=-2 L(\mathcal{G})+F(\mathcal{G})+2 V_{2}^{\prime}(\mathcal{G}) .
$$



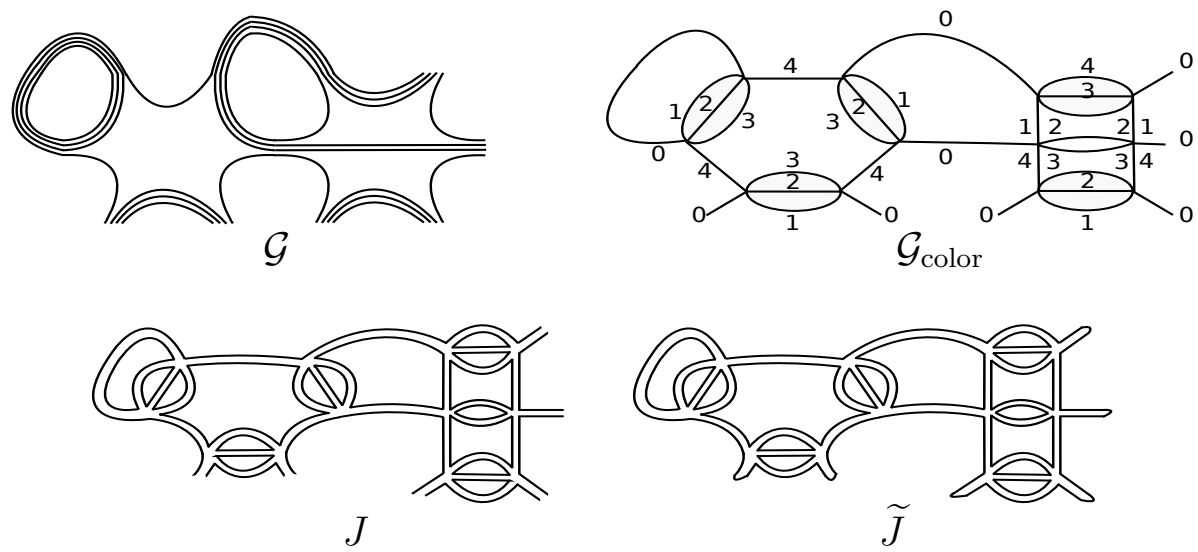

Figure 5: A graph $\mathcal{G}$, its colored extension $\mathcal{G}_{\text {color }}$ (five valence vertices with colored (half-)lines), the jacket subgraph $J$ (01234) of $\mathcal{G}_{\text {color }}$ and its associated pinched jacket $\widetilde{J}$.

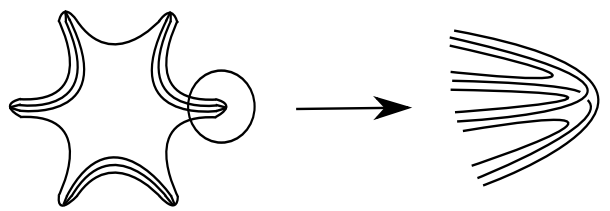

Figure 6: The boundary $\partial \mathcal{G}$ of $\mathcal{G}$ (see Fig 5 ) and its rank 3 tensor structure.

\section{Divergence Degree and Topology}

This section establishes another expression for the divergence degree $\omega_{d}\left(G_{i}^{(k)}\right)$ in an adequate form for the renormalization procedure. We will consider a general graph $\mathcal{G}$ rather than some $G_{i}^{(k)}$ and we introduce more ingredients for carrying through the analysis.

The following definitions follow the main ideas of jackets [33, 38, 39] and boundary graph 45.

Definition 1. Let $\mathcal{G}$ be a graph in our 4 dimensional theory.

(i) We call colored extension of $\mathcal{G}$ the unique graph $\mathcal{G}_{\text {color }}$ obtained after restoring in $\mathcal{G}$ the former colored theory graph (see Fig 5 ).

(ii) A jacket $J$ of $\mathcal{G}_{\text {color }}$ is a ribbon subgraph of $\mathcal{G}_{\text {color }}$ defined by a cycle $(0 a b c d)$ up to a cyclic permutation (see Fig[5). There are 12 such jackets in dimension 4 [39].

(iii) The jacket $\widetilde{J}$ is the jacket obtained from J after "pinching" viz. the procedure consisting in closing all external legs present in $J$ (see Fig.5). Hence it is always a vacuum graph.

(iv) The boundary $\partial \mathcal{G}$ of the graph $\mathcal{G}$ is the closed graph defined by vertices corresponding to external legs and by lines corresponding to external strands of $\mathcal{G}$ [45] (see Fig.6]). It is in our case a vacuum graph of the 3 dimensional colored theory.

(v) A boundary jacket $J_{\partial}$ is a jacket of $\partial \mathcal{G}$. There are 3 such boundary jackets in our case. 
Consider a connected graph $\mathcal{G}$. Let $V_{6}$ be its number of $\int \varphi^{6}$ type vertices (of any type) and $V_{4}$ its number of $\int \varphi^{4}$ vertices of the type $1, V_{4}^{\prime}$ its number of vertices of type $\left(\int \varphi^{2}\right)^{2}, V_{2}$ the number of vertices of the type $\int \varphi^{2}$ (mass counterterms) and $V_{2}^{\prime}$ the number of vertices of the type $\int(\nabla \varphi)^{2}$ (wave function counterterms). Let $L$ be its number of lines and $N_{\text {ext }}$ its number of external legs. Consider also its colored extension $\mathcal{G}_{\text {color }}$ and its boundary $\partial \mathcal{G}$.

Remark that the vertices contributing to $V_{4}^{\prime}$ are disconnected from the point of view of their strands. Hence it is convenient to reduce them in order to find the power counting with respect to only connected component graphs. We will consider these types of vertices as a pair of two 2-point vertices $V_{2}^{\prime \prime}$, hence $V_{2}^{\prime \prime}=2 V_{4}^{\prime}$. The vertices $V_{2}^{\prime \prime}$ are identical to the mass vertices $V_{2}$ except that they occur in pairs. The pairing is pictured in dotted line in Fig.3. The power counting can be established separately for each connected component after removing all the dotted lines.

The following statement, in the above notations, holds

Theorem 2. The divergence degree of a connected graph $\mathcal{G}$ is an integer which writes

$$
\omega_{d}(\mathcal{G})=-\frac{1}{3}\left[\sum_{J} g_{\widetilde{J}}-\sum_{J_{\partial}} g_{J_{\partial}}\right]-\left(C_{\partial \mathcal{G}}-1\right)-V_{4}-2\left(V_{2}+V_{2}^{\prime \prime}\right)-\frac{1}{2}\left[N_{\text {ext }}-6\right],
$$

where $g_{\widetilde{J}}$ and $g_{J_{\partial}}$ are the genus of $\widetilde{J}$ and $J_{\partial}$, respectively, $C_{\partial \mathcal{G}}$ is the number of connected components of the boundary graph $\partial \mathcal{G}$; the first sum is performed on all closed jackets $\widetilde{J}$ of $\mathcal{G}_{\text {color }}$ and the second sum is performed on all boundary jackets $J_{\partial}$ of $\partial \mathcal{G}$.

Proof. Given a connected graph (with respect to $V_{2}^{\prime \prime}$ and not to $V_{4}^{\prime}$ ) $\mathcal{G}$ with the above characteristics, we have the following relation between the numbers of lines, of external legs and of vertices:

$$
6 V_{6}+4 V_{4}+2\left(V_{2}+V_{2}^{\prime}+V_{2}^{\prime \prime}\right)=2 L+N_{\text {ext }} .
$$

Consider its colored extension $\mathcal{G}_{\text {color }}$. The latter graph is connected. Its number of vertices $V_{\mathcal{G}_{\text {color }}}$ and its number of lines $L_{\mathcal{G}_{\text {color }}}$ satisfy

$$
V_{\mathcal{G}_{\text {color }}}=6 V_{6}+4 V_{4}+2\left(V_{2}+V_{2}^{\prime}+V_{2}^{\prime \prime}\right), \quad L_{\mathcal{G}_{\text {color }}}=L+L_{\text {int } ; \mathcal{G}_{\text {color }}}=\frac{1}{2}\left(5 V_{\mathcal{G}_{\text {color }}}-N_{\text {ext }}\right),
$$

where $L_{\text {int } ; \mathcal{G}_{\text {color }}}$ are the internal lines of $\mathcal{G}_{\text {color }}$ which do not appear in $\mathcal{G}$. Let us denote $F_{\mathcal{G}_{\text {color }}}$ the number of faces of $\mathcal{G}_{\text {color }}$. The latter can be decomposed as well in terms of the number of faces of the initial graph, that is $F$, but also additional faces $F_{\text {int }} \mathcal{G}_{\text {color }}$ due to the internal colored structure:

$$
F_{\mathcal{G}_{\text {color }}}=F+F_{\text {int } ; \mathcal{G}_{\text {color }}} .
$$

Let us focus now on the 12 jackets of $\mathcal{G}_{\text {color }}$. Any such jacket is connected since $\mathcal{G}_{\text {color }}$ is connected. The following relations hold in the colored theory:

$$
V_{J}=V_{\mathcal{G}_{\text {color }}}, \quad L_{J}=L_{\mathcal{G}_{\text {color }}}, \quad N_{\text {ext } ; J}=N_{\text {ext } ; \mathcal{G}_{\text {color }}}=N_{\text {ext }} .
$$

Like the initial graph, a jacket may have open and closed faces. Each face of the graph $\mathcal{G}_{\text {color }}$ (open or closed) is shared by exactly $(D-1) !=6$ jackets. We have

$$
\sum_{J} F_{J}=6 F_{\mathcal{G}_{\text {color }}}
$$


The Euler characteristic of an open ribbon graph (i.e. a ribbon graph with external legs) is not well defined. Nevertheless, closing all external half-lines in a ribbon graph leads to another unique (closed) ribbon graph for which the above topological number is perfectly defined. This is the purpose of the pinching procedure applied to $J$ leading to $\widetilde{J}$. The resulting jacket $\widetilde{J}$ has the same number of vertices, the same number of lines as $J$, but a different number of faces than $J$. The number of faces of $\widetilde{J}$ can be partitioned into $F_{\widetilde{J}}=F_{\text {int } ; \widetilde{J}}+F_{\text {ext } ; \widetilde{J}}$, where $F_{\text {int } ; \widetilde{J}}$ corresponds to $F_{\text {int } ; J}$ the number of faces of $J$ and $F_{\text {ext } ; \widetilde{J}}$ is the number of additional closed faces created by the pinching procedure. Using the formula for the Euler characteristics of $\widetilde{J}$, we have

$$
F_{\text {int } ; \widetilde{J}}+F_{\text {ext } ; \widetilde{J}}=2-2 g_{\widetilde{J}}-V_{J}+L_{J} .
$$

Note that all external pinched faces $\widetilde{J}$ come from some open faces of the initial graph $\mathcal{G}$. However $F_{\text {int; } \widetilde{J}}$ can be decomposed in two categories of faces: one category of faces which belong to $\mathcal{G}$ (the number of such faces is denoted by $F_{\text {int; } \widetilde{J} ; \mathcal{G}}$ ) and another category of faces belonging only to the internal structure of $\mathcal{G}_{\text {color }}$ (the number of these latter faces is denoted

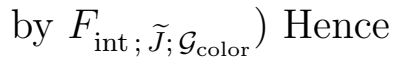

$$
F_{\text {int } ; \widetilde{J}}=F_{\text {int } ; \widetilde{J} ; \mathcal{G}}+F_{\text {int } ; \widetilde{J} ; \mathcal{G}_{\text {color }}} .
$$

The first step is to sum over all jackets in the l.h.s of 48):

$$
\sum_{J}\left(F_{\text {int } ; \widetilde{J} ; \mathcal{G}}+F_{\text {int } ; \widetilde{J} ; \mathcal{G}_{\text {color }}}+F_{\text {ext } ; \widetilde{J}}\right)=6 F_{\text {int } ; \mathcal{G}}+6 F_{\text {int } ; \mathcal{G}_{\text {color }}}+\sum_{J} F_{\text {ext } ; \widetilde{J}}
$$

Note that the number $F_{\text {int; }} \mathcal{G}_{\text {color }}$ of internal faces of $\mathcal{G}_{\text {color }}$ can be directly evaluated from any graph: each $\varphi^{6}$ vertex contains 12 such faces whereas each $\varphi^{4}$ vertex contains 9 and each $\varphi^{2}$ type vertices contains 6 internal faces so that

$$
F_{\text {int }} ; \mathcal{G}_{\text {color }}=12 V_{6}+9 V_{4}+6\left(V_{2}+V_{2}^{\prime}+V_{2}^{\prime \prime}\right) .
$$

Summing over all jackets the r.h.s. of (48), we focus on the following part:

$$
\sum_{J}\left[-V_{J}+L_{J}\right]=12\left[9 V_{6}+6 V_{4}+3\left(V_{2}+V_{2}^{\prime}+V_{2}^{\prime \prime}\right)\right]-6 N_{\mathrm{ext}} .
$$

Equating l.h.s and r.h.s, we extract the following relation for $F_{\text {int } ; \mathcal{G}}$ :

$$
F_{\text {int } ; \mathcal{G}}=-\frac{1}{6} \sum_{J} F_{\text {ext } ; \widetilde{J}}-\frac{1}{3} \sum_{J} g_{\widetilde{J}}+4+\left(6 V_{6}+3 V_{4}\right)-N_{\text {ext }} .
$$

The next stage is to re-express $\sum_{J} F_{\text {ext }: \widetilde{J}}$ in terms of topological numbers of the boundary graph of the graph $\mathcal{G}$. This boundary $\partial \mathcal{G}$ is defined such that

$$
V_{\partial \mathcal{G}}=N_{\mathrm{ext}}, \quad L_{\partial \mathcal{G}}=F_{\mathrm{ext}} .
$$

Since each external leg of the initial graph $\mathcal{G}$ has 4 strands and an external leg is made with two end-points belonging to two external legs, we have

$$
4 N_{\text {ext }}=2 F_{\text {ext }} .
$$




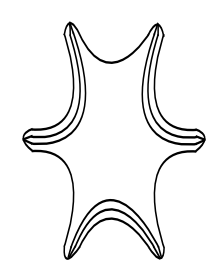

$\partial \mathcal{G}$

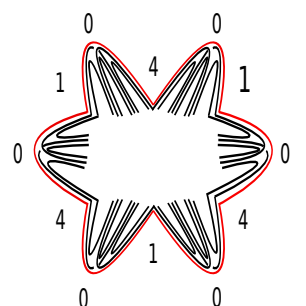

$f(014)$
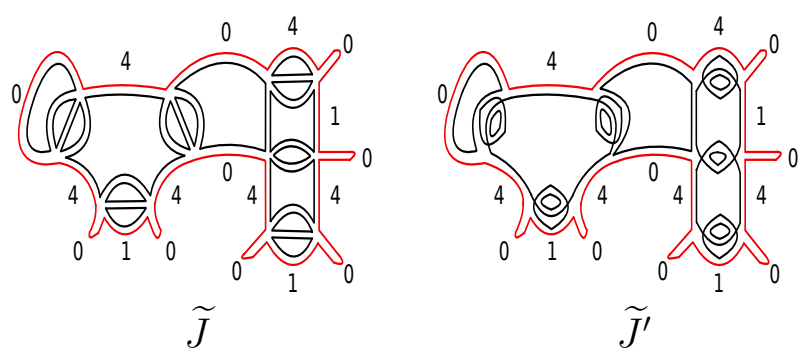

Figure 7: The boundary $\partial \mathcal{G}$ of graph $\mathcal{G}$ (see Fig 5 ), one of its colored face $f(014)$ (in red) and the unique two pinched jackets $\widetilde{J}(01234)$ and $\widetilde{J}^{\prime}(01324)$ of $\mathcal{G}_{\text {color }}$ containing $f$ (as highlighted).

The boundary graph is a closed (vacuum) colored graph living in the lower dimension $D-1=$ 3 . Hence, the boundary graph is again a tensor graph with jackets that will be denoted $J_{\partial}$. Remarkably, the boundary graph may be made of several connected components. The degree of $\partial \mathcal{G}$ is defined as the sum of genera of its jackets (which are all closed since $\partial \mathcal{G}$ is) which is $\omega_{\partial \mathcal{G}}=\sum_{J_{\partial}} g_{J_{\partial}}$, where $g_{J_{\partial}}$ is itself the sum of the genera of its connected components labeled by $\rho$, i.e. $g_{J_{\partial}}=\sum_{\rho} g_{J_{\partial}}$. Naturally, some relations on the numbers of vertices and lines between the boundary graph and jackets can be found:

$$
V_{J_{\partial}}=V_{\partial \mathcal{G}}=N_{\mathrm{ext}}, \quad L_{J_{\partial}}=L_{\partial \mathcal{G}}=F_{\mathrm{ext}} .
$$

Let $F_{J_{\partial}}$ the number of faces of $J_{\partial}$. The ordinary three dimensional colored relations apply to $J_{\partial}$ and $\partial \mathcal{G}$ :

$$
\sum_{J_{\partial}} F_{J_{\partial}}=(3-1) ! F_{\partial \mathcal{G}}=2 F_{\partial \mathcal{G}}, \quad \sum_{J_{\partial}} 1=\frac{1}{2} 3 !=3 .
$$

Thus, we have, using the Euler characteristic formula for boundary jackets,

$$
\sum_{J_{\partial}} F_{J_{\partial}}=\sum_{J_{\partial}}\left[\left(2 C_{J_{\partial}}-2 g_{J_{\partial}}\right)-V_{J_{\partial}}+L_{J_{\partial}}\right] \quad \Leftrightarrow \quad F_{\partial \mathcal{G}}=\sum_{J_{\partial}} C_{J_{\partial}}-\omega_{\partial \mathcal{G}}+\frac{3}{2} N_{\mathrm{ext}},
$$

where we restrict the study to the case $\partial \mathcal{G} \neq \emptyset$ so that $C_{J_{\partial}} \geq 1$. But $C_{J_{\partial}}=C_{\partial \mathcal{G}}$, then

$$
F_{\partial \mathcal{G}}=3\left(C_{\partial \mathcal{G}}-1\right)-\omega_{\partial \mathcal{G}}+3+\frac{3}{2} N_{\text {ext }} .
$$

On the other hand, consider the pinched jackets $\widetilde{J}$ of $\mathcal{G}_{\text {color }}$. From the fact that each face of the boundary graph $\partial \mathcal{G}$ (labeled by three colors, say $(0 a b)$ ) is shared by exactly 2 pinched jackets of the graph $\mathcal{G}_{\text {color }}$ (which will be labeled as $(0 a \ldots b)$ where the dots can be only the two remaining numbers $\check{a}, \breve{b} \in\{1,2,3,4\} \backslash\{a, b\}$; see Fig. 7 ),

we can relate

$$
\sum_{J} F_{\text {ext } ; \widetilde{J}}=2 F_{\partial \mathcal{G}}
$$

Inserting (59) into (60), then plugging the result in (53), furthermore, noting that $F_{\text {int } ; \mathcal{G}}=F$ and $2 L=6 V_{6}+4 V_{4}+2\left(V_{2}+V_{2}^{\prime}+V_{2}^{\prime \prime}\right)-N_{\text {ext }}$, the divergence degree $\omega_{d}(\mathcal{G})$ (41) can be recast 
in the form

$$
\begin{aligned}
\omega_{d}(\mathcal{G}) & =-2 L+F_{\text {int } ; \mathcal{G}}+2 V_{2}^{\prime} \\
& =-\frac{1}{3}\left[\left(3\left(C_{\partial \mathcal{G}}-1\right)-\omega_{\partial \mathcal{G}}+3+\frac{3}{2} N_{\text {ext }}\right)\right]-\frac{1}{3} \sum_{J} g_{\widetilde{J}}+4-V_{4}-2\left(V_{2}+V_{2}^{\prime \prime}\right) \\
& =-\frac{1}{3} \sum_{J} g_{\widetilde{J}}+\frac{1}{3} \sum_{J_{\partial}} g_{J_{\partial}}-\left(C_{\partial \mathcal{G}}-1\right)-V_{4}-2\left(V_{2}+V_{2}^{\prime \prime}\right)-\frac{1}{2}\left(N_{\text {ext }}-6\right)
\end{aligned}
$$

which is the desired relation.

The detailed analysis of the divergence degree is in order. An important part of that analysis is the understanding of the quantity

$$
-\frac{1}{3} \sum_{J} g_{\widetilde{J}}+\frac{1}{3} \sum_{J_{\partial}} g_{J_{\partial}}-\left(C_{\partial \mathcal{G}}-1\right) .
$$

This is purpose of the next section.

\section{Analysis of the Divergence Degree}

This section is divided in two parts: the first part addresses the study of the sign of the quantity 62 which is essential in the understanding of $\omega_{d}(\mathcal{G})$ and, based on this analysis, the second part classifies the primitively divergent graphs.

\subsection{Bounds on genera}

In this subsection, we restrict ourselves to the only important part of the problem, namely the analysis of graphs without any two-point $V_{2}, V_{2}^{\prime}$ and $V_{2}^{\prime \prime}$ vertices. Indeed, when such vertices are present, we can first contract any maximal chain of these vertices into a single line, then analyze the resulting reduced graph, then reintroduce the chains and the full graph analysis follows easily.

We now introduce a new tool to study of the divergence degree, namely a sequence of contractions which generalizes the idea of dipole contraction [38, 59, 60]. Contraction of dipoles separating bubbles in a colored theory can be performed without changing the degree. In particular a tree of 0-lines in a graph can always be contracted leading to a single "big" bubble with many 0-loops attached, which is the tensor analog of a rosette graph. This bubble is melonic if the initial vertices of the theory were melonic, which is the case here. In this subsection, we continue loop contractions on this generalized rosette which may change the degree. Hence, we generalize to the tensor context the analysis by Filk moves [61] of a ribbon rosette in non-commutative field theory [58].

Definition 2 (0k-dipole and contraction). We define a $0 k$-dipole, $k=0,1, \ldots, 4$, as a maximal subgraph of $\mathcal{G}_{\text {color }}$ made of $k+1$ lines joining two vertices, one of which of color 0 . Maximal means the $0 k$-dipole is not included in a $0(k+1)$-dipole.

The contraction of a $0 k$-dipole erases the $k+1$ lines of the dipole and joins the remaining $D-k$ lines on both sides of the dipole by respecting colors (see Fig.8). 


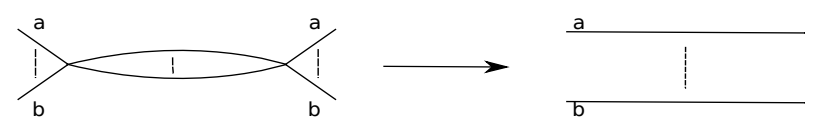

Figure 8: $0 k$-dipole contraction: external lines get glued.

Lemma 3 (Graph contraction). Performing the maximal number $\left(6 V_{6}+4 V_{4}-N_{\text {ext }}\right) / 2$ of $0 k$-dipole contractions on $\mathcal{G}_{\text {color }}$ in any arbitrary order and erasing the external legs of $\mathcal{G}$ leads to the boundary graph $\partial \mathcal{G}$.

Proof. The graph $\mathcal{G}_{\text {color }}$ possesses $V_{\mathcal{G}_{\text {color }}}=6 V_{6}+4 V_{4}$ vertices which can be decomposed in internal vertices $V_{\text {int } ; \mathcal{G}_{\text {color }}}=6 V_{6}+4 V_{4}-N_{\text {ext }}$ and external ones $N_{\text {ext }}$. To each internal vertices corresponds one half-line with color 0 , therefore $V_{\text {int } ; \mathcal{G}} / 2=L_{0 ; \text { int; } \mathcal{G}}$ is the number of lines of color 0 on which the contraction procedure will be applied.

Let us call the graph resulting from the contraction by $\widehat{\mathcal{G}}$. Since all vertices and lines of $\partial \mathcal{G}$ are not concerned by the procedure, $]^{7}$ they will appear again in $\widehat{\mathcal{G}}$ and hence, obviously, $\partial \mathcal{G} \subset \widehat{\mathcal{G}}$. Furthermore, given any order of contraction, the final graph has exactly the same number of vertices and lines than $\partial \mathcal{G}$. Therefore, these graphs should coincide: $\widehat{\mathcal{G}}=\partial \mathcal{G}$.

We turn now to the proof of two local lemmas which study the change in the sum over jackets of the difference in genera under a dipole contraction. We consider a colored connected graph $\mathcal{G}_{\text {color }}$, a fixed $0 k$-dipole and the contracted graph $\mathcal{G}_{\text {color }}^{\prime}$, which may or may not be connected. We notice first that during the contraction the numbers of vertices and lines change as

$$
V \rightarrow V^{\prime}=V-2, \quad L \rightarrow L^{\prime}=L-5,
$$

and the number of connected components can change from $c=1$ to $c^{\prime} \leq 4$. Note however that $c^{\prime}$ is constant for all jackets. Indeed, all jackets have the same number of connected components corresponding to the number of connected components of the graph obtained after contraction.

To track the change in faces, we introduce the notion of pair types for the contraction.

A pair for which the two colors are external to the dipole is called "outer". A pair which has one color inside the dipole and one out is called a "mixed" pair. A pair with two colors inside the dipole is called an "inner" pair. The total number of pairs is always 10 and the number of mixed pairs is at least 4. A pair is said to belong to a jacket if the pair is one of the five adjacent pairs in the jacket cycle.

We say that an outer pair is of type A, or disconnected by the dipole contraction if the half-strands at each corner on the left and on the right of the dipole belong to two different connected components of the graph after the dipole contraction. In the converse case, we call it a "special" pair. A special pair can be single-faced if the two corners belong to the same face of the graph, or double-faced if the two corners belong to two different faces of the graph. A moment of reflexion about open faces reveals that any type A outer pair must be single-faced at the beginning. Hence we have a classification of outer pairs into three types:

- Type A outer pairs are single-faced,

\footnotetext{
${ }^{7}$ For instance, an open face $(0 a)$ cannot be deleted by the dipole contraction procedure, it can just be shortened.
} 


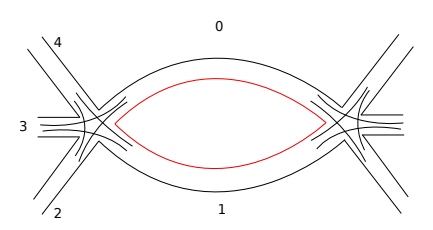

Figure 9: An inner face (in red) of a 01-dipole diagram.

- Type B outer pairs are single-faced,

- Type C outer pairs are double-faced.

Transverse pairs do not change their number of faces under contraction. Inner pairs have one face less after contraction. Type A and B outer pairs have one face more after contraction and type $\mathrm{C}$ outer pairs have one face less after the contraction. Hence for any jacket

$$
\left(F_{\widetilde{J}^{\prime}}-F_{\widetilde{J}}\right)=\left|A_{\widetilde{J}}\right|+\left|B_{\widetilde{J}}\right|-\left|C_{\widetilde{J}}\right|-\left|I_{\widetilde{J}}\right|,
$$

where $\left|A_{\widetilde{J}}\right|$ is the number of pairs of type $\mathrm{A}$ in the jacket and so on, and $\left|I_{\widetilde{J}}\right|$ is the number of inner faces (for an illustration, see Fig 9).

We prove now two lemmas ${ }^{8}$ analyzing the difference (64) after summing over jackets.

Lemma 4. Performing any $0 k$-dipole contraction on a graph, we obtain

$$
\sum_{J}\left(g_{\widetilde{J}}-g_{\widetilde{J}^{\prime}}\right) \geq 0
$$

Moreover, if $\sum_{J}\left(g_{\widetilde{J}}-g_{\widetilde{J}}\right)>0$ then

$$
\sum_{J}\left(g_{\widetilde{J}}-g_{\widetilde{J}^{\prime}}\right) \geq 6
$$

Proof. We have

$$
\begin{aligned}
2-2 g_{\widetilde{J}} & =V-L+F_{\widetilde{J}}, \\
2 c^{\prime}-2 g_{\widetilde{J}^{\prime}} & =V^{\prime}-L^{\prime}+F_{\widetilde{J}^{\prime}}=(V-2)-(L-5)+F_{\widetilde{J}^{\prime}}, \\
\left(g_{\widetilde{J}}-g_{\widetilde{J}^{\prime}}\right) & =\frac{1}{2}\left[\left(F_{\widetilde{J}^{\prime}}-F_{\widetilde{J}}\right)+3-2\left(c^{\prime}-1\right)\right] .
\end{aligned}
$$

Now using the fact that each face is shared by 6 jackets and that $c^{\prime}$ is constant for all jackets, by summing the last expression (67) over all jackets (recalling there are 12 of them) and using (64), we infer

$$
\sum_{J}\left(g_{\widetilde{J}}-g_{\widetilde{J}^{\prime}}\right)=3(A+B-C-I)+18-12\left(c^{\prime}-1\right)
$$

where we introduced the quantities $A=\sum_{J} A_{\widetilde{J}}, B=\sum_{J} B_{\widetilde{J}}, C=\sum_{J} C_{\widetilde{J}}$, and $I=\sum_{J} I_{\widetilde{J}}$.

\footnotetext{
${ }^{8}$ We warmly thank the referee for his constructive remarks improving the formulation and proof of these lemmas.
} 


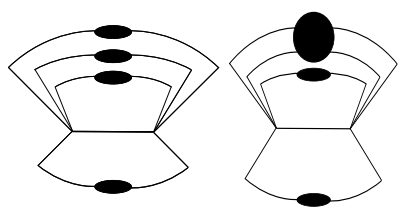

$7 \mathrm{~A}$

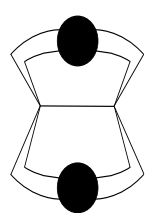

$7 \mathrm{Ca}$

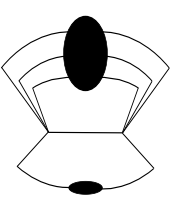

$7 \mathrm{Cb}$

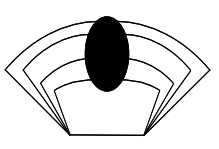

$7 \mathrm{D}$

Figure 10: 00-dipoles configurations.

We perform a case by case study, proving that $3(A+B-C-I)+18-12\left(c^{\prime}-1\right)$ is always positive and, always greater than 6 whenever it turns out to be strictly positive.

- 1rst Case: 00-dipole contraction. An unique internal line with color 0 is contracted. There are four mixed pairs, six outer pairs and no inner pair. Each jacket contains two mixed and three outer pairs.

- 1rst subcase $c^{\prime}=4$ (Fig. 7A). This can happen only if the resulting graph has on each line $1,2,3,4$, a connected two-point subgraph. In that case, all six outer pairs must be of type $A$. Hence, the 00-dipole contraction yields for all jackets:

$$
3(6)+18-12\left(c^{\prime}-1\right)=0 .
$$

- 2nd subcase $c^{\prime}=3$ (Fig. 7B). This case happens if we have two connected two-point functions plus one connected four-point function on four half-lines hooked to the dipole. In that case, we have 5 corner pairs of type A and one special pair, which can be type B or type C. We symbolically write for any possible choices:

$$
3(5 \pm 1)+18-12\left(c^{\prime}-1\right) \in\{6,12\} .
$$

- 3rd subcase $c^{\prime}=2$ (Fig. 7Ca and 7Cb). This can happen with two subsubcases: one with two connected four-point functions and one with one six-point and one two-point connected functions.

In the first subsubcase, there are 4 corner pairs of type A, and 2 of type either $\mathrm{B}$ or $\mathrm{C}$. We have

$$
3(4 \pm 1 \pm 1)+18-12\left(c^{\prime}-1\right) \in\{12,18,24\}
$$

In the second subsubcase, there are 3 outer pairs of type A and three special pairs. This corresponds to

$$
3(3 \pm 1 \pm 1 \pm 1)+18-12\left(c^{\prime}-1\right) \in\{6,12,18,24\} .
$$

- 4th subcase $c^{\prime}=1$ (Fig. 7D). Contracting the dipole gives a single connected component, hence $c^{\prime}-c=0$. It can happen if we have a eight-point connected function. There are no pairs of type A and six special pairs, hence

$$
3( \pm 1 \pm 1 \pm 1 \pm 1 \pm 1 \pm 1)+18-12\left(c^{\prime}-1\right) \in\{0,6,12,18,24,30,36\} .
$$

- 2nd Case: 01-dipole contraction. There is one inner pair, six mixed pairs and three outer pairs. There are three subcases.

- 1rst subcase $c^{\prime}=3$. In this case, the three outer pairs are type A, and there are no special pairs:

$$
3(3-1)+18-12\left(c^{\prime}-1\right)=0
$$


- 2nd subcase $c^{\prime}=2$. This situation yields two outer pairs of type A and one special:

$$
3(2 \pm 1-1)+18-12\left(c^{\prime}-1\right) \in\{6,12\} .
$$

- 3rd subcase $c^{\prime}=1$. Here, no outer pair is type A, the three outer pairs are special. This yields

$$
3( \pm 1 \pm 1 \pm 1-1)+18-12\left(c^{\prime}-1\right) \in\{6,12,18,24\} .
$$

- 3rd Case: 02-dipole contraction. There are three inner pairs, six mixed pairs and one outer pair. There are two subcases.

- 1rst subcase $c^{\prime}=2$. In this case, the outer pair is type A, and in all cases

$$
3(1-3)+18-12\left(c^{\prime}-1\right)=0 .
$$

- 2nd subcase $c^{\prime}=1$. Here, the outer pair is special and one gets

$$
3( \pm 1-3)+18-12\left(c^{\prime}-1\right) \in\{6,12\}
$$

- 4th Case: 03-dipole contraction. There are six inner pairs and four mixed pairs, $\left(c^{\prime}-1\right)=0$ such that one has

$$
3(-6)+18-12\left(c^{\prime}-1\right)=0 .
$$

- 5th Case: 04-dipole contraction. This is the easiest case as it destroys completely a full vacuum connected component with two vertices and five lines. In that case, there are ten inner pairs, $\left(c^{\prime}-1\right)=-1$ and so

$$
3(-10)+18-12\left(c^{\prime}-1\right)=0 .
$$

Hence in all above cases $(65)$ and $(66)$ are true.

Definition 3 (Jacket inclusion). We say that a 4-jacket $J$ (i.e. a jacket defined by a cycle of length 5 up to orientation) contains a 3-jacket $J^{\prime}$ without the color 0 , and we write $J^{\prime} \subset J$, if $J^{\prime}$ is the cycle obtained by contracting the color 0 is the cycle of $J$ (up to orientations).

There are obviously 4 jackets $J$, namely $(0 a b c d),(a 0 b c d),(a b 0 c d)$ and $(a b c 0 d)$ containing a given (boundary jacket) $J^{\prime}=(a b c d)$. They correspond to inserting 0 at any position in $J^{\prime}$.

Lemma 5 (Genus bounds). We have

$$
\sum_{J} g_{\widetilde{J}}-4 \sum_{J_{\partial}} g_{J_{\partial}} \in \mathbb{N}
$$

Moreover

$$
\begin{aligned}
\sum_{J_{\partial}} g_{J_{\partial}}>0 & \Rightarrow \sum_{J} g_{\widetilde{J}}-4 \sum_{J_{\partial}} g_{J_{\partial}} \geq 6, \\
\sum_{J_{\partial}} g_{J_{\partial}}=0 \text { and } \sum_{J} g_{\widetilde{J}}>0 & \Rightarrow \quad \sum_{J} g_{\widetilde{J}} \geq 6 .
\end{aligned}
$$


Proof. We perform a full sequence of $0 k$-dipole contractions on the initial graph $\mathcal{G}$ and arrive at the graph $\widehat{\mathcal{G}}=\partial \mathcal{G}$. By Lemma 4 , any genus of any pinched jacket $\widetilde{J}$ decreases along that sequence $9^{9}$ and, at the end, the pinched jacket coincides with the jacket $J_{\partial}$. To each boundary jacket $J_{\partial}$ we can associate four $\widetilde{J}$ such that $J_{\partial} \subset \widetilde{J}$. This proves (81)-(82). If $\sum_{J_{\partial}} g_{J_{\partial}}=0$ and $\sum_{J} g_{\widetilde{J}}>0$, then at some point along that sequence we can again use (66), which proves (83).

\subsection{Classification of divergent graphs}

We have $\partial \mathcal{G} \neq \emptyset$, hence $C_{J_{\partial}} \geq 1$, this means that we will always consider a graph $\mathcal{G}$ with a boundary in the following developments. Furthermore $C_{J_{\partial}} \leq N_{\text {ext }} / 2$, because each connected components must have at least a non zero even number of external legs. Let us define the integer $P(\mathcal{G})=\left(C_{\partial \mathcal{G}}-1\right)+V_{4}+2\left(V_{2}+V_{2}^{\prime \prime}\right)+\frac{1}{2}\left[N_{\text {ext }}-6\right]$. Lemma 5 translates into

Lemma 6 (Power counting bound). We have

$$
\begin{aligned}
\omega_{d}(\mathcal{G})=-\frac{1}{3}\left[\sum_{J} g_{\widetilde{J}}-\sum_{J_{\partial}} g_{J_{\partial}}\right]-P(\mathcal{G}) & \leq-\sum_{J_{\partial}} g_{J_{\partial}}-P(\mathcal{G}), \\
\sum_{J_{\partial}} g_{J_{\partial}}>0 & \Rightarrow \quad \omega_{d}(\mathcal{G}) \leq-2-\sum_{J_{\partial}} g_{J_{\partial}}-P(\mathcal{G}), \\
\sum_{J_{\partial}} g_{J_{\partial}}=0 \text { and } \sum_{J} g_{\widetilde{J}}>0 & \Rightarrow \quad \omega_{d}(\mathcal{G}) \leq-2-P(\mathcal{G}) .
\end{aligned}
$$

We search now for the list of graphs with $\omega_{d}(\mathcal{G}) \geq 0$ which are those which should be renormalized.

Case $N_{\text {ext }}>6$ : In this situation, $N_{\text {ext }} \geq 8$, so that $P(\mathcal{G}) \geq 1 \omega(\mathcal{G}) \leq-1$ and hence the graph has a converging amplitude.

Case $N_{\text {ext }}=6$ : The divergence degree is at most zero and can be so only if

$$
C_{J_{\partial}}=1, \quad \sum_{J_{\partial}} g_{J_{\partial}}=\sum_{J} g_{\widetilde{J}}=0, \quad V_{4}=V_{2}+V_{2}^{\prime \prime}=0 .
$$

Case $N_{\text {ext }}=4: P(\mathcal{G})=\left(C_{\partial \mathcal{G}}-1\right)+V_{4}+2\left(V_{2}+V_{2}^{\prime \prime}\right)-1$. The divergence degree is at most 1. It can be 1 only if $P(\mathcal{G})=-1$, and in fact if

$$
C_{J_{\partial}}=1, \quad \sum_{J_{\partial}} g_{J_{\partial}}=\sum_{J} g_{\widetilde{J}}=0, \quad V_{4}=V_{2}+V_{2}^{\prime \prime}=0 .
$$

But it could be zero if $P(\mathcal{G})=0$, in which case we must have either

$$
C_{J_{\partial}}=2, \quad \sum_{J_{\partial}} g_{J_{\partial}}=\sum_{J} g_{\widetilde{J}}=0, \quad V_{4}=V_{2}+V_{2}^{\prime \prime}=0
$$

\footnotetext{
${ }^{9}$ Given a jacket $\widetilde{J}$ and its contraction $\widetilde{J}^{\prime}$, we can also prove that $g_{\widetilde{J}} \geq g_{\widetilde{J}^{\prime}}$ using similar techniques as developed in Lemma 4. This inequality, holding jacket by jacket, is a stronger result than Lemma 4 .
} 


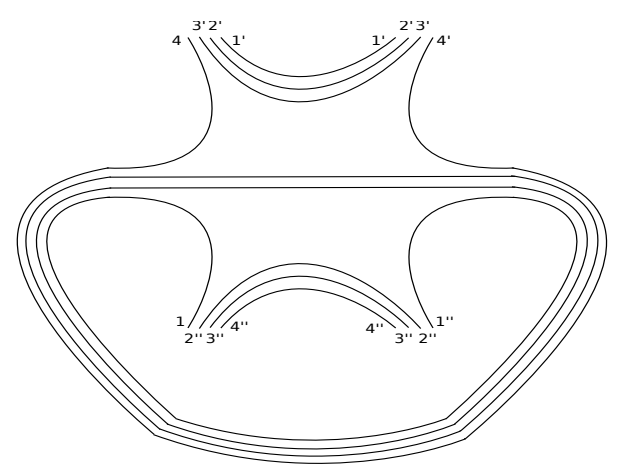

Figure 11: The tadpole of $V_{6 ; 2}$ has a disconnected boundary graph.

or

$$
C_{J_{\partial}}=1, \quad \sum_{J_{\partial}} g_{J_{\partial}}=\sum_{J} g_{\widetilde{J}}=0, \quad V_{4}=1, \quad V_{2}+V_{2}^{\prime \prime}=0 .
$$

Finally, when $P(\mathcal{G})=-1$, hence $C_{J_{\partial}}=1, V_{4}=V_{2}+V_{2}^{\prime \prime}=0$, if $\sum_{J_{\partial}} g_{J_{\partial}}>0$, we have $\omega_{d}(\mathcal{G}) \leq-2$ by 85 and if $\sum_{J_{\partial}} g_{J_{\partial}}=0$ and $\sum_{J} g_{\widetilde{J}}>0$ we have $\omega_{d}(\mathcal{G}) \leq-1$ by 86 .

Case $N_{\text {ext }}=2: P(\mathcal{G})=\left(C_{\partial \mathcal{G}}-1\right)+V_{4}+2\left(V_{2}+V_{2}^{\prime \prime}\right)-2$. In that case $\sum_{J_{\partial}} g_{J_{\partial}}=0$ since the only possible colored boundary graphs made with two external vertices is the standard one with six planar jackets. The analysis is slightly lengthy and we get 5 possible cases of divergent graphs.

In summary, the divergent graphs are determined by the following table:

\begin{tabular}{lccccc||c}
$N_{\text {ext }}$ & $V_{2}+V_{2}^{\prime \prime}$ & $V_{4}$ & $\sum_{J_{\partial}} g_{J_{\partial}}$ & $C_{\partial \mathcal{G}}-1$ & $\sum_{\widetilde{J}} g_{\widetilde{J}}$ & $\omega_{d}(\mathcal{G})$ \\
\hline \hline 6 & 0 & 0 & 0 & 0 & 0 & 0 \\
\hline 4 & 0 & 0 & 0 & 0 & 0 & 1 \\
4 & 0 & 1 & 0 & 0 & 0 & 0 \\
4 & 0 & 0 & 0 & 1 & 0 & 0 \\
\hline 2 & 0 & 0 & 0 & 0 & 0 & 2 \\
2 & 0 & 1 & 0 & 0 & 0 & 1 \\
2 & 0 & 2 & 0 & 0 & 0 & 0 \\
2 & 0 & 0 & 0 & 0 & 6 & 0 \\
2 & 1 & 0 & 0 & 0 & 0 & 0 \\
\hline \hline
\end{tabular}

Table 1

\subsection{The $\int \varphi^{2} \int \varphi^{2}$ anomalous term}

Since $V_{2}^{\prime \prime}$ is even, no graph with internal counterterm of the $V_{2}^{\prime \prime}$ can appear in the previous table. However the fourth row of the table corresponds to melonic graphs with disconnected boundary graphs, they do appear and are really divergent. The first and lowest order example example is the special "diagonal tadpole" built on the $V_{6 ; 2}$ vertex (see Fig 11). This graph has one line and two internal faces $0 a$ and $0 b$ where $a$ and $b$ are the colors of the two "inner strands" in the $V_{6 ; 2}$ interaction. Hence $-2 L+F=0$. The graph is really logarithmically 
divergent as the propagator $1 /\left(p^{2}+m^{2}\right)$ is positive, hence there is no way any unexpected cancellation could affect its amplitude.

It is difficult to interpret yet this anomalous term but it happens in 4 dimensions and not in 3. This kind of factorized log-divergent term is best represented as an integral over an intermediate field as

$$
e^{-\left(\int \varphi^{2}\right)^{2}}=c \int d \sigma e^{-\int \sigma^{2}-2 i \int \sigma \varphi^{2}}
$$

$c$ being some constant, and whose propagator is the dotted line in Fig.3. It joins the two two-point functions. But this propagator does not have any strand, hence our gravity theory generates a scalar matter field.

\section{Renormalization}

We now implement the renormalization program for the $p$-point functions which are divergent and characterized as given by Table 1. We use Taylor expansions around the local parts in direct space in the manner of [55, 62].

\subsection{Renormalization of the six-point function}

Consider a general six-point function subgraph $G_{i}^{(k)}$, namely with $N_{\text {ext }}\left(G_{i}^{(k)}\right)=6$ of the type of the first line of Table 1 . Since $\sum_{J_{\partial}} g_{J_{\partial}}=0$, we know that the boundary graph is itself a melonic graph and hence the pattern of external positions follows the form either of $V_{6 ; 1}$ or of $V_{6 ; 2}$.

We reintroduce the graph with external propagators and call its amplitude $\bar{A}_{6}\left(G_{i}^{(k)}\right)$. External positions variables are labeled by $\theta_{l, s}^{\text {ext }}, l=1,2,3,4,5,6$ and $s=1,2,3$, 4 , where $s$ is, as usually, the strand index while $l$ can be considered as the external leg index with scale $j_{l}$. Recall that $j_{l}$ indices are strictly smaller than $i$ the index of $G_{i}^{(k)}$. $\theta_{l, s}^{0}$ denotes the position connected to the external end-point $\theta_{l, s}^{\text {ext }}$.

The following procedure is standard [55] and consists in performing a Taylor expansion in direct space by interpolating moves of the external legs. Remark that this interpolation should be periodic and consistent with the fact that we are dealing with a torus. For convenience, we also change the local parametrization and integration bounds of the Haar measure to be $[-\pi, \pi)$. Hence, we interpolate $\theta_{l(v), s}^{0}$ using a parameter $t \in[0,1]$ such that, for $\theta_{l^{\prime}\left(v^{\prime}\right), s}^{0} \in[0, \pi)$

$$
\begin{aligned}
\theta_{l(v), s}^{0} \in\left[\theta_{l^{\prime}\left(v^{\prime}\right), s}^{0}-\pi, \pi\right), & \theta_{l(v), s}^{0}=\theta_{l^{\prime}\left(v^{\prime}\right), s}^{0}+\left.t\left(\theta_{l(v), s}^{0}-\theta_{l^{\prime}\left(v^{\prime}\right), s}^{0}\right)\right|_{t=1}, \\
\theta_{l(v), s}^{0} \in\left[-\pi, \theta_{l^{\prime}\left(v^{\prime}\right), s}^{0}-\pi\right), & \theta_{l(v), s}^{0}=\theta_{l^{\prime}\left(v^{\prime}\right), s}^{0}-2 \pi+\left.t\left(\theta_{l(v), s}^{0}-\theta_{l^{\prime}\left(v^{\prime}\right), s}^{0}+2 \pi\right)\right|_{t=1},
\end{aligned}
$$

where $\theta_{l^{\prime}\left(v^{\prime}\right), s}^{0}$ is the internal position connected to the external index $\theta_{l^{\prime}, s}^{\text {ext }}$ which can be associated with $\theta_{l, s}^{\text {ext }}$ according to the particular pattern of the $\varphi^{6}$ vertices. From the above, one can easily infer the interpolation for the other range of values $\theta_{l^{\prime}\left(v^{\prime}\right), s}^{0} \in[-\pi, 0)$.

We relate the lines $l$ and $l^{\prime}$ and their strand index $s$ in two possible ways dictated by the boundary graphs of a $\varphi^{6}$ form: 
(1) the couples $\left(\theta_{l, s}^{0}, \theta_{l^{\prime}, s}^{0}\right)$ for $\left(l, l^{\prime}\right) \in\{(2,1),(4,3),(6,5)\}$ are connected with respect to the strand indices $s=2,3,4$, whereas pairs $\left(l, l^{\prime}\right) \in\{(1,6),(5,4),(3,2)\}$ will be connected only for the strand index $s=1$; performing a permutation on the role of $s=1,2,3$ and 4 gives the parametrization for remainder vertices of $V_{6 ; 1}$;

(2) the couples $\left(\theta_{l, s}^{0}, \theta_{l^{\prime}, s}^{0}\right)$ for $\left(l, l^{\prime}\right) \in\{(3,2),(6,5)\}$ are connected for strand indices $s=$ $2,3,4,\left(l, l^{\prime}\right)=(4,1)$ connected for $s=2,3,\left(l, l^{\prime}\right) \in\{(2,1),(4,3)\}$ are connected for a single index $s=4$ and $\left(l, l^{\prime}\right) \in\{(5,4),(1,6)\}$ are connected for $s=1$; a permutation on the role of $(2,3)$ for any other couple in $\{1,2,3,4\}$ yields the parameterizations for remainder vertices as defined by $V_{6 ; 2}$.

In the following, we will focus on the vertex of the first kind defined by pairings (1). For the second kind, it can be checked that similar results will be also valid.

Consider the amplitude of the subgraph $G_{i}^{(k)}$ with external propagators characterized as above given by (in simplified notations)

$$
\begin{aligned}
\bar{A}_{6}\left(G_{i}^{(k)}\right)\left[\left\{\theta_{l, s}^{\mathrm{ext}}\right\}\right]= & \int\left\{\left[\prod_{\ell} d \theta_{\ell, s}\right]\left[\prod_{l} C_{j_{l}}\left(\left\{\theta_{l, s}^{\mathrm{ext}}\right\} ;\left\{\theta_{l(v), s}^{0}\right\}\right)\right]\right. \\
& {\left.\left[\prod_{\ell \neq l} C_{i_{\ell}}\left(\left\{\theta_{\ell(v), s}\right\} ;\left\{\theta_{\ell\left(v^{\prime}\right), s}\right\}\right)\right] \prod_{v \in \mathcal{V}} \delta\left(\theta_{v, s}-\theta_{v, s^{\prime}}\right)\right\} . }
\end{aligned}
$$

We introduce a function $\bar{A}_{6}\left(G_{i}^{(k)}\right)\left[\left\{\theta_{l, s}^{\text {ext }}\right\} ; t\right]$ and write the amplitude $\sqrt[93]{ }$ as $\bar{A}_{6}\left(G_{i}^{(k)}\right)\left[\left\{\theta_{l, s}^{\text {ext }}\right\}\right.$; $t=1]=\bar{A}_{6}\left(G_{i}^{(k)}\right)\left[\left\{\theta_{l, s}^{\text {ext }}\right\} ; t=0\right]+\int_{0}^{1} d t \frac{d}{d t} \bar{A}_{6}\left(G_{i}^{(k)}\right)\left[\left\{\theta_{l, s}^{\text {ext }}\right\} ; t\right]$, where the function $\bar{A}_{6}\left(G_{i}^{(k)}\right)\left[\left\{\theta_{l, s}^{\text {ext }}\right\} ; t\right]$ is obtained by interpolating (93) using (92), namely

$$
\begin{aligned}
\bar{A}_{6}\left(G_{i}^{(k)}\right)\left[\left\{\theta_{l, s}^{\mathrm{ext}}\right\} ; t\right]= & \int\left[\prod_{\ell} d \theta_{\ell, s}\right]\left\{\left[\prod_{l=1}^{6} C_{j_{l}}\left(\left\{\theta_{l, s}^{\mathrm{ext}}\right\} ;\left\{\theta_{l^{\prime}\left(v^{\prime}\right), s}^{0}+t\left(\theta_{l(v), s}^{0}-\theta_{l^{\prime}\left(v^{\prime}\right), s}^{0}\right)\right\}\right)\right]\right. \\
& {\left.\left[\prod_{\ell \neq l} C_{i_{\ell}}\left(\left\{\theta_{\ell(v), s}\right\} ;\left\{\theta_{\ell\left(v^{\prime}\right), s}\right\}\right)\right] \prod_{v \in \mathcal{V}} \delta\left(\theta_{v, s}-\theta_{v, s^{\prime}}\right)\right\} . }
\end{aligned}
$$

Henceforth, $\left\{\theta_{l^{\prime}\left(v^{\prime}\right), s}^{0}+t\left(\theta_{l(v), s}^{0}-\theta_{l^{\prime}\left(v^{\prime}\right), s}^{0}\right)\right\}$ denotes any formula of the periodic interpolation (92) according to the range of values of the coordinates.

The term at $t=0$ is given by

$$
\begin{aligned}
& \bar{A}_{6}\left(G_{i}^{(k)}\right)\left[\left\{\theta_{l, s}^{\text {ext }}\right\} ; 0\right]=\int\left\{\left[\prod_{\ell \neq l} d \theta_{\ell, s}\right]\left[\prod_{l=1}^{6} C_{j_{l}}\left(\left\{\theta_{l, s}^{\text {ext }}\right\} ;\left\{\theta_{l^{\prime}\left(v^{\prime}\right), s}^{0}\right\}\right)\right]\right. \\
& \left.\int\left[\left[\prod_{l=2 p+1} d \theta_{l, 1}^{0}\right]\left[\prod_{l=2 p} d \theta_{l, 2}^{0} d \theta_{l, 3}^{0} d \theta_{l, 4}^{0}\right]\right]\left[\prod_{\ell \neq l} C_{i_{\ell}}\left(\left\{\theta_{\ell(v), s}\right\} ;\left\{\theta_{\ell\left(v^{\prime}\right), s}\right\}\right)\right] \prod_{v \in \mathcal{V}} \delta\left(\theta_{v, s}-\theta_{v, s^{\prime}}\right)\right\} \\
& \prod_{l=1}^{6} C_{j_{l}}\left(\left\{\theta_{l, s}^{\text {ext }}\right\} ;\left\{\theta_{l^{\prime}\left(v^{\prime}\right), s}^{0}\right\}\right)= \\
& C_{j_{1}}\left(\left\{\theta_{1, s}^{\text {ext }}\right\} ;\left\{\theta_{6,1}^{0}, \theta_{1,2}^{0}, \theta_{1,3}^{0}, \theta_{1,4}^{0}\right\}\right) C_{j_{2}}\left(\left\{\theta_{2, s}^{\text {ext }}\right\} ;\left\{\theta_{2,1}^{0}, \theta_{1,2}^{0}, \theta_{1,3}^{0}, \theta_{1,4}^{0}\right\}\right) \\
& C_{j_{3}}\left(\left\{\theta_{3, s}^{\text {ext }}\right\} ;\left\{\theta_{2,1}^{0}, \theta_{3,2}^{0}, \theta_{3,3}^{0}, \theta_{3,4}^{0}\right\}\right) C_{j_{4}}\left(\left\{\theta_{4, s}^{\text {ext }}\right\} ;\left\{\theta_{4,1}^{0}, \theta_{3,2}^{0}, \theta_{3,3}^{0}, \theta_{3,4}^{0}\right\}\right) \\
& C_{j_{5}}\left(\left\{\theta_{5, s}^{\text {ext }}\right\} ;\left\{\theta_{4,1}^{0}, \theta_{5,2}^{0}, \theta_{5,3}^{0}, \theta_{5,4}^{0}\right\}\right) C_{j_{6}}\left(\left\{\theta_{6, s}^{\text {ext }}\right\} ;\left\{\theta_{6,1}^{0}, \theta_{5,2}^{0}, \theta_{6,3}^{0}, \theta_{6,4}^{0}\right\}\right),
\end{aligned}
$$


where we remove the possible $2 \pi$ coming from the interpolation using the periodicity of the functions. Next, the remainder finds the following expansion

$$
\begin{aligned}
R_{6}= & \int_{0}^{1} d t \int\left\{[ \prod _ { \ell } d \theta _ { \ell , s } ] \left(\sum_{l=1}^{6}\left[\prod_{p \neq l} C_{j_{p}}\left(\left\{\theta_{p, s}^{\mathrm{ext}}\right\} ;\left\{\theta_{p^{\prime}\left(v^{\prime}\right), s}^{0}+t\left(\theta_{p(v), s}^{0}-\theta_{p^{\prime}\left(v^{\prime}\right), s}^{0}\right)\right\}\right)\right]\right.\right. \\
& \left.T_{l} \triangleright C_{j_{l}}\left(\left\{\theta_{l s}^{\mathrm{ext}}\right\} ;\left\{\theta_{l^{\prime}\left(v^{\prime}\right), s}^{0}+t\left(\theta_{l(v), s}^{0}-\theta_{l^{\prime}\left(v^{\prime}\right), s}^{0}\right)\right\}\right)\right) \\
& {\left.\left[\prod_{\ell \neq l} C_{i_{\ell}}\left(\left\{\theta_{\ell(v), s}\right\} ;\left\{\theta_{\ell\left(v^{\prime}\right), s}\right\}\right)\right] \prod_{v \in \mathcal{V}} \delta\left(\theta_{v, s}-\theta_{v, s^{\prime}}\right)\right\}, }
\end{aligned}
$$

where the operator $T_{l}$ differentiates with respect to particular strands according to the vertex pattern and is given by

$$
T_{l}=\sum_{k=0}^{3}\left[\delta_{l, 2 k+1}\left(\theta_{l(v), 1}^{0}-\theta_{l^{\prime}\left(v^{\prime}\right), 1}^{0}+r\right) \partial_{\theta ; 1}+\delta_{l, 2 k} \sum_{s=2}^{4}\left(\theta_{l(v), s}^{0}-\theta_{l^{\prime}\left(v^{\prime}\right), s}^{0}+r\right) \partial_{\theta ; s}\right],
$$

where $\partial_{\theta ; s}$ is a partial derivative with respect to the second set of arguments of $C_{j_{l}}$ containing $\theta^{0}$ 's and taken at the strand $s$ and $r= \pm 2 \pi$ or 0 according to the sector of interpolation. It remains to analyze these terms. Considering (95), the following statement holds

Lemma 7. The quantity

$$
\int\left[\left[\prod_{l=2 p+1} d \theta_{l, 1}^{0}\right]\left[\prod_{l=2 p} d \theta_{l, 2}^{0} d \theta_{l, 3}^{0} d \theta_{l, 4}^{0}\right]\right]\left[\prod_{\ell \neq l} C_{i_{\ell}}\left(\left\{\theta_{\ell(v), s}\right\} ;\left\{\theta_{\ell\left(v^{\prime}\right), s}\right\}\right)\right] \prod_{v \in \mathcal{V}} \delta\left(\theta_{v, s}-\theta_{v, s^{\prime}}\right)
$$

does not depends on $\left\{\theta_{l^{\prime}, s}^{0}\right\}$ defined by $\prod_{l=1}^{6} C_{i_{l}}\left(\left\{\theta_{l, s}^{\text {ext }}\right\} ;\left\{\theta_{l^{\prime}\left(v^{\prime}\right), s}^{0}\right\}\right)$.

Proof. This is a consequence of translation invariance of the propagators in spatial coordinates that we now review quickly. Having performed a Taylor expansion of the interpolated amplitude $(94)$, the zeroth order term is of the form $\sqrt{95}$, where the set arguments $\left\{\theta_{l^{\prime}\left(v^{\prime}\right), s}^{0}\right\}$ present in the product $\prod_{l=1}^{6} C_{i_{l}}\left(\left\{\theta_{l, s}^{\text {ext }}\right\} ;\left\{\theta_{l^{\prime}\left(v^{\prime}\right), s}^{0}\right\}\right)$ may be still involved in the internal structure. For simplicity, we focus on $\theta_{6,1}^{0}$ and we can consider the external face formed by successive positions $\theta_{1,1}^{0}, \theta_{l_{1}, s_{1}}, \theta_{l_{2}, s_{2}}, \ldots, \theta_{l_{q}, s_{q}}, \theta_{6,1}^{0}$. The propagators generating the face amplitude associated with this sequence are functions of the differences $\left(\theta_{l_{\alpha}, s_{\alpha}}-\theta_{l_{\beta}, s_{\beta}}\right)$. Since $\theta_{1,1}^{0}$ and $\theta_{6,1}^{0}$ are external end-points, we can always perform a change of variable $\widetilde{\theta}_{l_{\beta}, s_{\beta}}=\theta_{l_{\alpha}, s_{\alpha}}-\theta_{l_{\beta}, s_{\beta}}$ to remove one of these external position labels. Note that, since we are dealing with a compact space, the bounds of integration of the new variables $\widetilde{\theta}_{l_{\alpha}, s_{\alpha}}$ change. Nonetheless, recall that the propagators here are periodic so that all these integration bounds can be translated indifferently to $[-\pi, \pi)$. In the present situation, choosing to remove $\theta_{1,1}^{0}$, we obtain a face amplitude independent of that variable. Reproducing the argument for each external faces, one proves the lemma.

Lemma 8. The remainder $R_{6}$ of the amplitude interpolation can be bounded by

$$
\left|R_{6}\right| \leq K M^{-\left(i\left(G_{i}^{(k)}\right)-e\left(G_{i}^{(k)}\right)\right)} M^{\omega\left(G_{i}^{(k)}\right)}, \quad e\left(G_{i}^{(k)}\right)=\sup _{\text {lexternal to } G_{i}^{(k)}} j_{l}, \quad i\left(G_{i}^{(k)}\right)=\inf _{l \in G_{i}^{(k)}} i_{l},
$$

for some constant $K$. 
Proof. Let us first make a remark concerning the integration bounds due to the splitting introduced by the interpolation which is in the rough form (focusing on $r=+2 \pi$ )

$$
\int_{0}^{\pi} d \theta_{l^{\prime}, s}^{0} \int_{\theta_{l^{\prime}, s}^{0}-\pi}^{\pi} d \theta_{l, s}^{0}\left(\theta_{l(v), s}^{0}-\theta_{l^{\prime}\left(v^{\prime}\right), s}^{0}\right) \prod C+\int_{0}^{\pi} d \theta_{l^{\prime}, s}^{0} \int_{-\pi}^{\theta_{l^{\prime}, s}^{0}-\pi} d \theta_{l, s}^{0}\left(\theta_{l(v), s}^{0}-\theta_{l^{\prime}\left(v^{\prime}\right), s}^{0}+2 \pi\right) \prod C
$$

We can perform a change of variable in the term $\hat{\theta}_{l(v), s}^{0}=\theta_{l(v), s}^{0}+2 \pi$ for which, clearly, the products of covariances and delta functions remain invariant such that

$$
\int_{0}^{\pi} d \theta_{l^{\prime}, s}^{0} \int_{\theta_{l^{\prime}, s}^{0}-\pi}^{\pi} d \theta_{l, s}^{0}\left(\theta_{l(v), s}^{0}-\theta_{l^{\prime}\left(v^{\prime}\right), s}^{0}\right) \prod C+\int_{0}^{\pi} d \theta_{l^{\prime}, s}^{0} \int_{\pi}^{\theta_{l^{\prime}, s}^{0}+\pi} d \hat{\theta}_{l, s}^{0}\left(\hat{\theta}_{l(v), s}^{0}-\theta_{l^{\prime}\left(v^{\prime}\right), s}^{0}\right) \prod C .
$$

By summing the two internal integrals we get a single integral as $\int_{\theta^{\prime}-\pi}^{\theta^{\prime}+\pi} d \theta_{l, s}^{0}\left(\theta_{l(v), s}^{0}-\theta_{l^{\prime}\left(v^{\prime}\right), s}^{0}\right)$. What we have gained here is that the final integral can be fully bounded in terms of the difference $\left(\theta_{l(v), s}^{0}-\theta_{l^{\prime}\left(v^{\prime}\right), s}^{0}\right)$.

Lemma 1 yields a bound on the first derivative of the propagator (24) as

$$
\partial_{\theta, s} C_{j_{l}}\left(\left\{\theta_{s^{\prime}}^{\text {ext }}\right\} ;\left\{\Theta_{s^{\prime}}\right\}\right) \leq K M^{3 j_{l}} e^{-\delta M^{j_{l}} \sum_{s^{\prime}}\left|\theta_{s^{\prime}}^{\text {ext }}-\Theta_{s^{\prime}}\right|}
$$

so that, taking the best estimate between external scales, the following bound holds

$$
\begin{aligned}
&\left|R_{6}\right| \leq K^{\prime} M^{e\left(G_{i}^{(k)}\right)} M^{-i\left(G_{i}^{(k)}\right)} \int_{0}^{1} d t \int\left\{[ \prod _ { \ell } d \theta _ { \ell , s } ] \left(\sum _ { l = 1 } ^ { 6 } \left[\prod_{p \neq l} M^{-2 j_{l}} e^{\left.-\delta M^{j_{p}} \sum_{s} \mid \theta_{p, s}^{\mathrm{ext}}-\theta_{p^{\prime}\left(v^{\prime}\right), s}^{0}\right]}\right.\right.\right. \\
&\left.\left.M^{2 e\left(G_{i}^{(k)}\right)} e^{-\delta M^{j_{l}} \sum_{s^{\prime}}\left|\theta_{l, s}^{\mathrm{ext}}-\theta_{l^{\prime}\left(v^{\prime}\right), s}^{0}\right|}\right)\left[\prod_{\ell \neq l} C_{i_{\ell}}\left(\left\{\theta_{\ell(v), s}\right\} ;\left\{\theta_{\ell\left(v^{\prime}\right), s}\right\}\right)\right] \prod_{v \in \mathcal{V}} \delta\left(\theta_{v, s}-\theta_{v, s^{\prime}}\right)\right\},
\end{aligned}
$$

we have used the facts that high internal decays entail $\left|\left(\theta_{l(v), s}^{0}-\theta_{l^{\prime}\left(v^{\prime}\right), s}^{0}\right)\right| \sim M^{-i_{l}}$ and hence, $\left|\theta_{p, s}^{\text {ext }}-\left(\theta_{p^{\prime}\left(v^{\prime}\right), s}^{0}+t\left(\theta_{p(v), s}^{0}-\theta_{p^{\prime}\left(v^{\prime}\right), s}^{0}\right)\right)\right| \sim\left|\theta_{p, s}^{\text {ext }}-\theta_{p^{\prime}\left(v^{\prime}\right), s}^{0}\right|$ and, also, that the distance between the two internal positions, say $\theta_{l(v), s}^{0}$ and $\theta_{l^{\prime}\left(v^{\prime}\right), s}^{0}$, can be optimized by choosing $\left|\theta_{l(v), s}^{0}-\theta_{l^{\prime}\left(v^{\prime}\right), s}^{0}\right| \leq$ $M^{-i\left(G_{i}^{(k)}\right)}$. As an effect, the integral in $t$ factors and we get the result. 7)

In conclusion, we have found that the zeroth order counterterm is given by (using Lemma

$$
\bar{A}_{6}\left(G_{i}^{(k)}\right)\left[\left\{\theta_{l, s}^{\mathrm{ext}}\right\} ; 0\right]=\log M \int\left[\prod_{l^{\prime}} d \theta_{l^{\prime}, s}\right] \prod_{l=1}^{6} C_{j_{l}}\left(\left\{\theta_{l, s}^{\mathrm{ext}}\right\} ;\left\{\theta_{l^{\prime}, s}^{0}\right\}\right)
$$

hence is of the form vertex $V_{6 ; 1}\left[{ }^{10}\right.$ and is logarithmically divergent, whereas the sub-leading term is actually convergent due the power counting improvement by $M^{-\left(i\left(G_{i}^{(k)}\right)-e\left(G_{i}^{(k)}\right)\right)}$. This is exactly what is needed in order to perform the sum over the momentum assignments. One can easily check that performing the similar analysis to other kind of permuted vertices $V_{6 ; 1}$ or $V_{6 ; 2}$ will lead to the same result.

\footnotetext{
${ }^{10}$ In fact, this a vertex $V_{6 ; 1}$ with six external propagator integrated to it.
} 


\subsection{Renormalization of the four-point function}

We use the same procedure as above in order to find the counterterms of the four-point function and for that consider a four-point function subgraph $G_{i}^{(k)}$, characterized by the one of the three lines of Table 1. Three cases may occur but, in all situations, the graph (which should be melonic with a melonic boundary graph in all cases) has an external structure either of the form $V_{4 ; 1}$ or of the form $V_{4 ; 2}$. The latter class includes graphs with disconnected boundary graph (the last line of the table for $N_{\text {ext }}=4$ ).

Let us call $\bar{A}_{4}\left(G_{i}^{(k)}\right)$ the amplitude associated with $G_{i}^{(k)}$ equipped with external propagators. External position variables are labeled by $\theta_{l, s}^{\text {ext }}, l=1,2,3,4$ and $s=1,2,3,4$ and external legs are at scale $j_{l}$. We keep the same meaning of $\theta_{l, s}^{0}$ as the positions connected to the external end-points $\theta_{l, s}^{\text {ext }}$.

Interpolating $\theta_{l(v), s}^{0}$ using $(92)$, according to the particular pattern of external positions of the boundary graph of the $\varphi^{4}$ type, we have:

(1) the couples $\left(\theta_{l, s}^{0}, \theta_{l^{\prime}, s}^{0}\right)$ for $\left(l, l^{\prime}\right) \in\{(2,1),(4,3)\}$ are connected with respect to the strand indices $s=2,3,4$, whereas pairs $\left(l, l^{\prime}\right) \in\{(1,4),(3,2)\}$ will be connected only for the strand index $s=1$; performing a permutation on the role of $s=1,2,3$ and 4 gives the parametrization for remainder vertices of $V_{4 ; 1}$;

(2) the couples $\left(\theta_{l, s}^{0}, \theta_{l^{\prime}, s}^{0}\right)$ for $\left(l, l^{\prime}\right) \in\{(1,2),(3,4)\}$ are connected for all strand indices $s=1,2,3,4$ and this defines the pattern of $V_{6 ; 2}$.

Once again, we will only focus on the vertex of the first kind (1) since the same reasoning will be valid for any other cases. The amplitude $\bar{A}_{4}\left(G_{i}^{(k)}\right)\left[\left\{\theta_{l, s}^{\text {ext }}\right\}\right]$ of the subgraph $G_{i}^{(k)}$ with external propagators (with above characteristics) is given by a formula similar to (93), and using external leg interpolations giving the parametrized amplitude $\bar{A}_{4}\left(G_{i}^{(k)}\right)\left[\left\{\theta_{l, s}^{\text {ext }}\right\} ; t\right]$, we write

$$
\begin{aligned}
& \bar{A}_{4}\left(G_{i}^{(k)}\right)\left[\left\{\theta_{l, s}^{\text {ext }}\right\} ; t=1\right]= \\
& \bar{A}_{4}\left(G_{i}^{(k)}\right)\left[\left\{\theta_{l, s}^{\text {ext }}\right\} ; t=0\right]+\frac{d}{d t} \bar{A}_{4}\left(G_{i}^{(k)}\right)\left[\left\{\theta_{l, s}^{\text {ext }}\right\} ; t=0\right]+\int_{0}^{1} d t(1-t) \frac{d^{2}}{d t^{2}} \bar{A}_{4}\left(G_{i}^{(k)}\right)\left[\left\{\theta_{l, s}^{\text {ext }}\right\} ; t\right],
\end{aligned}
$$

where the function $\bar{A}_{4}\left(G_{i}^{(k)}\right)\left[\left\{\theta_{l, s}^{\text {ext }}\right\} ; t\right]$ is given by a quantity analog to $(94)$, with four external propagators. At $t=0$, we get the contribution

$$
\begin{aligned}
& \bar{A}_{4}\left(G_{i}^{(k)}\right)\left[\left\{\theta_{l, s}^{\text {ext }}\right\} ; 0\right]=\int\left\{\left[\prod_{\ell \neq l} d \theta_{\ell, s}\right]\left[\prod_{l=1}^{4} C_{j_{l}}\left(\left\{\theta_{l, s}^{\text {ext }}\right\} ;\left\{\theta_{l^{\prime}\left(v^{\prime}\right), s}^{0}\right\}\right)\right]\right. \\
& \left.\int\left[\left[\prod_{l=2 p+1} d \theta_{l, 1}^{0}\right]\left[\prod_{l=2 p} d \theta_{l, 2}^{0} d \theta_{l, 3}^{0} d \theta_{l, 4}^{0}\right]\right]\left[\prod_{\ell \neq l} C_{i_{\ell}}\left(\left\{\theta_{\ell(v), s}\right\} ;\left\{\theta_{\ell\left(v^{\prime}\right), s}\right\}\right)\right] \prod_{v \in \mathcal{V}} \delta\left(\theta_{v, s}-\theta_{v, s^{\prime}}\right)\right\} ; \\
& \prod_{l=1}^{4} C_{j_{l}}\left(\left\{\theta_{l s}^{\text {ext }}\right\} ;\left\{\theta_{l^{\prime}\left(v^{\prime}\right), s}^{0}\right\}\right)= \\
& C_{j_{1}}\left(\left\{\theta_{1, s}^{\text {ext }}\right\} ;\left\{\theta_{4,1}^{0}, \theta_{1,2}^{0}, \theta_{1,3}^{0}, \theta_{1,4}^{0}\right\}\right) C_{j_{2}}\left(\left\{\theta_{2, s}^{\text {ext }}\right\} ;\left\{\theta_{2,1}^{0}, \theta_{1,2}^{0}, \theta_{1,3}^{0}, \theta_{1,4}^{0}\right\}\right) \\
& C_{j_{3}}\left(\left\{\theta_{3, s}^{\text {ext }}\right\} ;\left\{\theta_{2,1}^{0}, \theta_{3,2}^{0}, \theta_{3,3}^{0}, \theta_{3,4}^{0}\right\}\right) C_{j_{4}}\left(\left\{\theta_{4, s}^{\text {ext }}\right\} ;\left\{\theta_{4,1}^{0}, \theta_{3,2}^{0}, \theta_{3,3}^{0}, \theta_{3,4}^{0}\right\}\right) .
\end{aligned}
$$


The second term is given by

$$
\begin{aligned}
& \frac{d}{d t} \bar{A}_{4}\left(G_{i}^{(k)}\right)\left[\left\{\theta_{l, s}^{\mathrm{ext}}\right\} ; t=0\right]=\int\left\{[ \prod _ { \ell } d \theta _ { \ell , s } ] \left(\sum_{l=1}^{6}\left[\prod_{p \neq l} C_{j_{p}}\left(\left\{\theta_{p, s}^{\mathrm{ext}}\right\} ;\left\{\theta_{p^{\prime}\left(v^{\prime}\right), s}^{0}\right\}\right)\right]\right.\right. \\
& \left.\left.T_{l} \triangleright C_{j_{l}}\left(\left\{\theta_{l, s}^{\mathrm{ext}}\right\} ;\left\{\theta_{l^{\prime}\left(v^{\prime}\right), s}^{0}\right\}\right)\right)\left[\prod_{\ell \neq l} C_{i_{\ell}}\left(\left\{\theta_{\ell(v), s}\right\} ;\left\{\theta_{\ell\left(v^{\prime}\right), s}\right\}\right)\right] \prod_{v \in \mathcal{V}} \delta\left(\theta_{v, s}-\theta_{v, s^{\prime}}\right)\right\},
\end{aligned}
$$

where the operator $T_{l}$ now refers to

$$
T_{l}=\sum_{k=0}^{2}\left[\delta_{l, 2 k+1}\left(\theta_{l(v), 1}^{0}-\theta_{l^{\prime}\left(v^{\prime}\right), 1}^{0}+r\right) \partial_{\theta ; 1}+\delta_{l, 2 k} \sum_{s=2}^{4}\left(\theta_{l(v), s}^{0}-\theta_{l^{\prime}\left(v^{\prime}\right), s}^{0}+r\right) \partial_{\theta ; s}\right],
$$

with $\partial_{\theta ; s}$ and $r$ keeping their sense as in (97). Finally and in the same anterior notations, the remainder computes to

$$
\begin{aligned}
& R_{4}=\int_{0}^{1} d t(1-t) \int\left[\prod_{\ell} d \theta_{\ell, s}\right]\{ \\
& \left(\sum _ { l = 1 } ^ { 6 } \left[\sum_{l^{\prime} \neq l}\left[\prod_{q \neq l^{\prime}} C_{j_{q}}\left(\left\{\theta_{q, s}^{\text {ext }}\right\} ;\left\{\theta_{q^{\prime}\left(v^{\prime}\right), s}^{0}+t\left(\theta_{q(v), s}-\theta_{q^{\prime}\left(v^{\prime}\right), s}\right)\right\}\right)\right]\right.\right. \\
& \times T_{l^{\prime}} C_{j_{l^{\prime}}}\left(\left\{\theta_{l^{\prime} s}^{\text {ext }}\right\} ;\left\{\theta_{l^{\prime \prime}\left(v^{\prime}\right), s}^{0}+t\left(\theta_{l^{\prime}(v), s}^{0}-\theta_{l^{\prime \prime}\left(v^{\prime}\right), s}^{0}\right)\right\}\right) \\
& \times T_{l} \triangleright C_{j_{l}}\left(\left\{\theta_{l, s}^{\text {ext }}\right\} ;\left\{\theta_{l^{\prime}\left(v^{\prime}\right), s}^{0}+t\left(\theta_{l(v), s}^{0}-\theta_{l^{\prime}\left(v^{\prime}\right), s}^{0}\right)\right\}\right) \\
& +\left[\prod_{p \neq l} C_{j_{p}}\left(\left\{\theta_{p, s}^{\text {ext }}\right\} ;\left\{\theta_{p^{\prime}\left(v^{\prime}\right), s}^{0}+t\left(\theta_{p(v), s}-\theta_{p^{\prime}\left(v^{\prime}\right), s}\right)\right\}\right)\right] \\
& \left.\left.T_{l} \triangleright T_{l} \triangleright C_{j_{l}}\left(\left\{\theta_{l s}^{\text {ext }}\right\} ;\left\{\theta_{l^{\prime}\left(v^{\prime}\right), s}^{0}+t\left(\theta_{l(v), s}^{0}-\theta_{l^{\prime}\left(v^{\prime}\right), s}^{0}\right)\right\}\right)\right]\right) \\
& \left.\left[\prod_{\ell \neq l} C_{i_{\ell}}\left(\left\{\theta_{\ell(v), s}\right\} ;\left\{\theta_{\ell\left(v^{\prime}\right), s}\right\}\right)\right] \prod_{v \in \mathcal{V}} \delta\left(\theta_{v, s}-\theta_{v, s^{\prime}}\right)\right\} .
\end{aligned}
$$

The following statement holds

Lemma 9. The internal contribution of $\bar{A}_{4}\left(G_{i}^{(k)}\right)\left[\left\{\theta_{l, s}^{\text {ext }}\right\} ; 0\right]$, namely

$$
\int\left[\left[\prod_{l=2 p+1} d \theta_{l, 1}^{0}\right]\left[\prod_{l=2 p} d \theta_{l, 2}^{0} d \theta_{l, 3}^{0} d \theta_{l, 4}^{0}\right]\right]\left[\prod_{\ell \neq l} C_{i_{\ell}}\left(\left\{\theta_{\ell(v), s}\right\} ;\left\{\theta_{\ell\left(v^{\prime}\right), s}\right\}\right)\right] \prod_{v \in \mathcal{V}} \delta\left(\theta_{v, s}-\theta_{v, s^{\prime}}\right)
$$

does not depends on the set of variables $\left\{\theta_{l^{\prime}\left(v^{\prime}\right), s}^{0}\right\}$ used in the interpolation moves. Furthermore, the second contribution identically vanishes:

$$
\frac{d}{d t} \bar{A}_{4}\left(G_{i}^{(k)}\right)\left[\left\{\theta_{l, s}^{e x t}\right\} ; 0\right]=0 .
$$

Proof. The first claim can be proved using translation invariance along the lines of the proof of Lemma 7. Indeed, the main point here is that, once again, one of the external position on external faces can be absorbed by successive changes of variables along a face. We simply choose to gauge away the interpolated positions belonging to $\left\{\theta_{l^{\prime}, s}^{0}\right\}$. 
The second claim can be proved using the parity of functions. To this end, we start by writing the said contribution as

$$
\begin{aligned}
& \frac{d}{d t} \bar{A}_{4}\left(G_{i}^{(k)}\right)\left[\left\{\theta_{l, s}^{\mathrm{ext}}\right\} ; 0\right]= \\
& \int\left\{[ \prod _ { \ell } d \theta _ { \ell , s } ] \left(\sum_{l=1}^{4}\left[\prod_{p \neq l} C_{j_{p}}\left(\left\{\theta_{p, s}^{\mathrm{ext}}\right\} ;\left\{\theta_{p^{\prime}\left(v^{\prime}\right), s}^{0}\right\}\right)\right]\right.\right. \\
& \left.\sum_{k=0}^{2}\left[\delta_{l, 2 k+1}\left(\theta_{l, 1}^{0}-\theta_{l^{\prime}, 1}^{0}+r\right) \partial_{\theta ; 1}+\delta_{l, 2 k} \sum_{s=2}^{4}\left(\theta_{l, s}^{0}-\theta_{l^{\prime}, s}^{0}+r\right) \partial_{\theta ; s}\right] C_{j_{l}}\left(\left\{\theta_{l, s}^{\mathrm{ext}}\right\} ;\left\{\theta_{l^{\prime}\left(v^{\prime}\right), s}^{0}\right\}\right)\right) \\
& \left.\left[\prod_{\ell \neq l} C_{i_{\ell}}\left(\left\{\theta_{\ell(v), s}\right\} ;\left\{\theta_{\ell\left(v^{\prime}\right), s}\right\}\right)\right] \prod_{v \in \mathcal{V}} \delta\left(\theta_{v, s}-\theta_{v, s^{\prime}}\right)\right\} .
\end{aligned}
$$

One notices that the internal contribution

$$
\int\left[\prod_{\ell} d \theta_{\ell, s}\right]\left(\theta_{l, s}^{0}-\theta_{l^{\prime}, s}^{0}+r\right)\left[\prod_{\ell \neq l} C_{i_{\ell}}\left(\left\{\theta_{\ell(v), s}\right\} ;\left\{\theta_{\ell\left(v^{\prime}\right), s}\right\}\right)\right] \prod_{v \in \mathcal{V}} \delta\left(\theta_{v, s}-\theta_{v, s^{\prime}}\right)
$$

does not depend on $\left\{\theta_{l^{\prime}, s}^{0}\right\}$ by translation invariance and factors from the external data. We make two successive change of variables such that, $\hat{\theta}_{l, s}^{0}=\theta_{l, s}^{0}+r$, in all corresponding sectors of the theory, and then, for all lines $\ell,\left(\theta_{\ell, s}-\theta_{l^{\prime}, s}^{0}\right)=\tilde{\theta}_{\ell, s}^{0}$, and the internal part becomes

$$
\begin{aligned}
& \int_{-\pi}^{\pi} d \tilde{\theta}_{l, s}^{0} \tilde{\theta}_{l, s}^{0} C_{i_{l}}\left(\left\{\tilde{\theta}_{l, s}^{0} ; \tilde{\theta}_{l(v), s}\right\} ;\left\{\tilde{\theta}_{l\left(v^{\prime}\right), s}\right\}\right) \\
& \int\left[\prod_{\ell \neq l} d \theta_{\ell, s}\right]\left[\prod_{\ell \neq l} C_{i_{\ell}}\left(\left\{\tilde{\theta}_{\ell(v), s}\right\} ;\left\{\tilde{\theta}_{\ell\left(v^{\prime}\right), s}\right\}\right)\right] \prod_{v \in \mathcal{V}} \delta\left(\tilde{\theta}_{v, s}-\tilde{\theta}_{v, s^{\prime}}\right) .
\end{aligned}
$$

The result of this integral is vanishing due to the parity of all propagators (see (21)) and delta functions while $\tilde{\theta}_{l, s}^{0}$ is clearly odd.

Lemma 10. The remainder $R_{4}$ of the amplitude interpolation can be bounded by

$$
\left|R_{4}\right| \leq K M^{-2\left(i\left(G_{i}^{(k)}\right)-e\left(G_{i}^{(k)}\right)\right)} M^{\omega\left(G_{i}^{(k)}\right)}, \quad e\left(G_{i}^{(k)}\right)=\sup _{\text {lexternal to } G_{i}^{(k)}} j_{l}, \quad i\left(G_{i}^{(k)}\right)=\inf _{l \in G_{i}^{(k)}} i_{l},
$$

for some constant $K$.

Proof. The proof starts by removing all $r$ in the same manner as performed in the proof of Lemma 8 using the periodicity of all kernels. Then, expanding the derivative in the propagators of the $T^{2}$ form, we can bound the second order products as $\mid\left(\theta_{l_{1}, s_{1}}^{0}-\theta_{l_{1}^{\prime}, s_{1}}^{0}\right)\left(\theta_{l_{2}, s_{2}}^{0}-\right.$ $\left.\theta_{l_{2}^{\prime}, s_{2}}^{0}\right) \mid \leq M^{-2 i\left(G_{i}^{(k)}\right)}$ whereas each derivative $\partial_{\theta ; s} C_{j_{l}}$ by 24 yields a factor $M^{e\left(G_{i}^{(k)}\right)}$ (second order derivative will contribute twice, and so forth). We collect these improvements and write, using internal decay to remove the differences $t\left(\theta_{l, s}^{0}-\theta_{l^{\prime}, s}^{0}\right)$ and dropping the integral in $t$,

$$
\left|R_{4}\right| \leq K M^{2 e\left(G_{i}^{(k)}\right)} M^{-2 i\left(G_{i}^{(k)}\right)} \int\left[\prod_{\ell} d \theta_{\ell, s}\right]\{
$$




$$
\begin{aligned}
& \left(\sum_{l=1}^{6}\left[\prod_{q \neq l} M^{2 j_{q}} e^{-\delta M^{j q} \sum_{s} \mid \theta_{q, s}^{\mathrm{ext}}-\theta_{q^{\prime}\left(v^{\prime}\right), s}^{0}}\right] M^{2 e\left(G_{i}^{(k)}\right)} e^{-\delta M^{j_{l}} \sum_{s}\left|\theta_{l, s}^{\mathrm{ext}}-\theta_{l^{\prime}\left(v^{\prime}\right), s}^{0}\right|}\right) \\
& \left.\left[\prod_{\ell \neq l} C_{i_{\ell}}\left(\left\{\theta_{\ell(v), s}\right\} ;\left\{\theta_{\ell\left(v^{\prime}\right), s}\right\}\right)\right] \prod_{v \in \mathcal{V}} \delta\left(\theta_{v, s}-\theta_{v, s^{\prime}}\right)\right\} .
\end{aligned}
$$

At this stage, we have proved that the local part of amplitude is linear and of the form of the initial vertex $V_{4 ; 1}$. For the second kind of vertex appearing $V_{4 ; 2}$ and permutations, the same analysis also applies.

\subsection{Renormalization of the two-point function}

We perform now the interpolation moves for external legs of the two-point function of a subgraph $G_{i}^{(k)}$ defined by the one of the five lines of Table 1 . Here, we will be dealing with a graph with boundary of the form of a mass type vertex of the kind $V_{2}$.

Let $\bar{A}_{2}\left(G_{i}^{(k)}\right)$ denote the amplitude associated with $G_{i}^{(k)}$ equipped with external propagators with external positions variables $\theta_{l, s}^{\text {ext }}, l=1,2$, and $s=1,2,3,4$, and scale $j_{l}$. The couples $\left(\theta_{l, s}^{0}, \theta_{l, s}^{\text {ext }}\right)$ keep their earlier relationship and sense.

We use the formula (92) in order to rewrite $\theta_{2, s}^{0}$ according to the particular pattern of the $\varphi^{2}$ vertices: the couples $\left(\theta_{1, s}^{0}, \theta_{2, s}^{0}\right)$ are connected with respect to the strand indices $s=1,2,3,4$.

The amplitude $\bar{A}_{2}\left(G_{i}^{(k)}\right)\left[\left\{\theta_{l, s}^{\text {ext }}\right\}\right]$ of the subgraph $G_{i}^{(k)}$ with external propagators (with above characteristics) is re-expressed using the modified amplitude $\bar{A}_{2}\left(G_{i}^{(k)}\right)\left[\left\{\theta_{l, s}^{\text {ext }}\right\} ; t\right]$ as

$$
\begin{aligned}
\bar{A}_{2}\left(G_{i}^{(k)}\right)\left[\left\{\theta_{l, s}^{\text {ext }}\right\} ; t=1\right] & =\bar{A}_{2}\left(G_{i}^{(k)}\right)\left[\left\{\theta_{l, s}^{\text {ext }}\right\} ; t=0\right]+\frac{d}{d t} \bar{A}_{2}\left(G_{i}^{(k)}\right)\left[\left\{\theta_{l, s}^{\text {ext }}\right\} ; t=0\right] \\
& +\frac{1}{2} \frac{d^{2}}{d t^{2}} \bar{A}_{2}\left(G_{i}^{(k)}\right)\left[\left\{\theta_{l, s}^{\text {ext }}\right\} ; t=0\right]+\frac{1}{2} \int_{0}^{1}(1-t)^{2} \frac{d^{3}}{d t^{3}} \bar{A}_{2}\left(G_{i}^{(k)}\right)\left[\left\{\theta_{l, s}^{\text {ext }}\right\} ; t\right]
\end{aligned}
$$

where we define

$$
\begin{aligned}
\bar{A}_{2}\left(G_{i}^{(k)}\right)\left[\left\{\theta_{l, s}^{\text {ext }}\right\} ; t\right]= & \int\left[\prod_{\ell} d \theta_{\ell, s}\right]\left\{C_{j_{1}}\left(\left\{\theta_{1 s}^{\text {ext }}\right\} ;\left\{\theta_{1, s}^{0}\right\}\right) C_{j_{2}}\left(\left\{\theta_{2, s}^{\text {ext }}\right\} ;\left\{\theta_{1, s}^{0}+t\left(\theta_{2, s}^{0}-\theta_{1, s}^{0}\right)\right\}\right)\right. \\
& {\left.\left[\prod_{\ell \neq l} C_{i_{\ell}}\left(\left\{\theta_{\ell(v), s}\right\} ;\left\{\theta_{\ell\left(v^{\prime}\right), s}\right\}\right)\right] \prod_{v \in \mathcal{V}} \delta\left(\theta_{v, s}-\theta_{v, s^{\prime}}\right)\right\} }
\end{aligned}
$$

The different quantities involved in the expansion can be studied. The first contribution is of the form

$$
\begin{aligned}
& \bar{A}_{2}\left(G_{i}^{(k)}\right)\left[\left\{\theta_{l, s}^{\mathrm{ext}}\right\} ; 0\right]=\int\left\{\left[\prod_{\ell \neq l} d \theta_{\ell, s}\right]\left[\prod_{l=1}^{2} C_{j_{l}}\left(\left\{\theta_{l, s}^{\mathrm{ext}}\right\} ;\left\{\theta_{l^{\prime}\left(v^{\prime}\right), s}^{0}\right\}\right)\right]\right. \\
& \left.\int\left[\prod_{s} d \theta_{2, s}^{0}\right]\left[\prod_{\ell \neq l} C_{i_{\ell}}\left(\left\{\theta_{\ell(v), s}\right\} ;\left\{\theta_{\ell\left(v^{\prime}\right), s}\right\}\right)\right] \prod_{v \in \mathcal{V}} \delta\left(\theta_{v, s}-\theta_{v, s^{\prime}}\right)\right\} \\
& \prod_{l=1}^{2} C_{j_{l}}\left(\left\{\theta_{l, s}^{\mathrm{ext}}\right\} ;\left\{\theta_{l^{\prime}\left(v^{\prime}\right), s}^{0}\right\}\right)=
\end{aligned}
$$




$$
C_{j_{1}}\left(\left\{\theta_{1, s}^{\text {ext }}\right\} ;\left\{\theta_{1,1}^{0}, \theta_{1,2}^{0}, \theta_{1,3}^{0}, \theta_{1,4}^{0}\right\}\right) C_{j_{2}}\left(\left\{\theta_{2, s}^{\text {ext }}\right\} ;\left\{\theta_{1,1}^{0}, \theta_{1,2}^{0}, \theta_{1,3}^{0}, \theta_{1,4}^{0}\right\}\right) .
$$

The $\alpha$-th derivative terms, $\alpha=1,2$, are given by

$$
\begin{aligned}
\frac{d^{\alpha}}{d t^{\alpha}} \bar{A}_{2}\left(G_{i}^{(k)}\right)\left[\left\{\theta_{l, s}^{\text {ext }}\right\} ; t=0\right]= & \int\left\{\left[\prod_{\ell} d \theta_{\ell, s}\right]\left(C_{j_{1}}\left(\left\{\theta_{1, s}^{\text {ext }}\right\} ;\left\{\theta_{1, s}^{0}\right\}\right)\right] T^{\alpha} \triangleright C_{j_{2}}\left(\left\{\theta_{2, s}^{\text {ext }}\right\} ;\left\{\theta_{1, s}^{0}\right\}\right)\right) \\
& {\left.\left[\prod_{\ell \neq l} C_{i_{\ell}}\left(\left\{\theta_{\ell(v), s}\right\} ;\left\{\theta_{\ell\left(v^{\prime}\right), s}\right\}\right)\right] \prod_{v \in \mathcal{V}} \delta\left(\theta_{v, s}-\theta_{v, s^{\prime}}\right)\right\}, \quad(120) }
\end{aligned}
$$

where $T^{\alpha}$ stands for the operator, using previous notations,

$$
T^{\alpha}:=\sum_{s_{\alpha}} \prod_{\alpha}\left(\theta_{2, s_{\alpha}}^{0}-\theta_{1, s_{\alpha}}^{0}+r\right) \prod_{\alpha} \partial_{\theta ; s_{\alpha}}, \quad \alpha=1,2,3, \quad s_{\alpha}=1,2,3,4
$$

Last, the remainder can be written as

$$
\begin{aligned}
R_{2}= & \frac{1}{2} \int_{0}^{1} d t(1-t)^{2} \int\left[\prod_{\ell} d \theta_{\ell, s}\right]\{ \\
& \left.\left(C_{j_{1}}\left(\left\{\theta_{1, s}^{\text {ext }}\right\} ;\left\{\theta_{1, s}^{0}\right\}\right)\right] T^{3} \triangleright C_{j_{2}}\left(\left\{\theta_{2, s}^{\text {ext }}\right\} ;\left\{\theta_{1, s}^{0}+t\left(\theta_{2, s}^{0}-\theta_{1, s}^{0}\right)\right\}\right)\right) \\
& {\left.\left[\prod_{\ell \neq l} C_{i_{\ell}}\left(\left\{\theta_{\ell(v), s}\right\} ;\left\{\theta_{\ell\left(v^{\prime}\right), s}\right\}\right)\right] \prod_{v \in \mathcal{V}} \delta\left(\theta_{v, s}-\theta_{v, s^{\prime}}\right)\right\} }
\end{aligned}
$$

The main properties of the different parts of the expansion are summarized in the following propositions.

Lemma 11. The internal contribution of $\bar{A}_{2}\left(G_{i}^{(k)}\right)\left[\left\{\theta_{l, s}^{\text {ext }}\right\} ; 0\right]$, namely

$$
\int\left[\left[\prod_{s} d \theta_{2, s}^{0}\right]\left[\prod_{\ell \neq l} C_{i_{\ell}}\left(\left\{\theta_{\ell(v), s}\right\} ;\left\{\theta_{\ell\left(v^{\prime}\right), s}\right\}\right)\right] \prod_{v \in \mathcal{V}} \delta\left(\theta_{v, s}-\theta_{v, s^{\prime}}\right)\right.
$$

does not depends on the set of variables $\left\{\theta_{1, s}^{0}\right\}$ used in the interpolation moves. Furthermore, we have

$$
\frac{d}{d t} \bar{A}_{2}\left(G_{i}^{(k)}\right)\left[\left\{\theta_{l, s}^{e x t}\right\} ; 0\right]=0
$$

and the third term reduces to

$$
\frac{d^{2}}{d t^{2}} \bar{A}_{2}\left(G_{i}^{(k)}\right)\left[\left\{\theta_{l, s}^{e x t}\right\} ; 0\right]=\log M \int\left[\prod_{s} d \theta_{1, s}\right] C_{j_{1}}\left(\left\{\theta_{1, u}^{e x t}\right\} ;\left\{\theta_{1, u}^{0}\right\}\right) \sum_{s=1}^{4} \Delta_{s}^{2} C_{j_{2}}\left(\left\{\theta_{2, v}^{e x t}\right\} ;\left\{\theta_{1, v}^{0}\right\}\right)
$$

where $\Delta_{s}$ is a Laplace operator on $U(1)$ acting on the strand $s$.

Proof. The first claim is a consequence of translation invariance and provides a mass renormalization. The second claim can be proved using the parity of propagators along the lines of the proof of Lemma 9. Let us focus on the last claim and write (using more symbols in order to differentiate strand indices)

$$
\frac{d^{2}}{d t^{2}} \bar{A}_{2}\left(G_{i}^{(k)}\right)\left[\left\{\theta_{l, s}^{\text {ext }}\right\} ; t=0\right]=
$$




$$
\begin{aligned}
& \sum_{s, s^{\prime}=1}^{4} \int\left\{\left[\prod_{s} d \theta_{1, s}\right]\left(C_{j_{1}}\left(\left\{\theta_{1, u}^{\text {ext }}\right\} ;\left\{\theta_{1, u}^{0}\right\}\right) \partial_{\theta ; s} \partial_{\theta ; s^{\prime}} C_{j_{2}}\left(\left\{\theta_{2, w}^{\text {ext }}\right\} ;\left\{\theta_{1, w}^{0}\right\}\right)\right)\right. \\
& \int\left[\prod_{\ell \neq l} d \theta_{\ell, s}\right]\left[\left(\theta_{2, s}^{0}-\theta_{1, s}^{0}+r\right)\left(\theta_{2, s^{\prime}}^{0}-\theta_{1, s^{\prime}}^{0}+r\right) \prod_{\ell \neq l} C_{i_{\ell}}\left(\left\{\theta_{\ell(v), q}\right\} ;\left\{\theta_{\ell\left(v^{\prime}\right), q}\right\}\right)\right] \\
& \left.\prod_{v \in \mathcal{V}} \delta\left(\theta_{v, s}-\theta_{v, s^{\prime}}\right)\right\} .
\end{aligned}
$$

We first implement a change of variable which removes all $r$. Next, we use translation invariance in order to remove from the internal part the dependence in $\theta_{1, s}^{0}$. One gets

$$
\int\left[\prod_{\ell \neq 1} d \tilde{\theta}_{\ell, s}\right]\left[\tilde{\theta}_{2, s}^{0} \tilde{\theta}_{2, s^{\prime}}^{0} \prod_{\ell \neq l} C_{i_{\ell}}\left(\left\{\tilde{\theta}_{\ell(v), s}\right\} ;\left\{\tilde{\theta}_{\ell\left(v^{\prime}\right), s}\right\}\right)\right] \prod_{v \in \mathcal{V}} \delta\left(\tilde{\theta}_{v, s}-\tilde{\theta}_{v, s^{\prime}}\right) .
$$

When $s \neq s^{\prime}$, the above integral vanishes because of the parity of the integrand function, recall that $\tilde{\theta}_{2, s}^{0}$ and $\tilde{\theta}_{2, s^{\prime}}^{0}$ belong to $[-\pi, \pi)$. Only remains the terms at $s=s^{\prime}$ which are

$$
\begin{aligned}
& \frac{d^{2}}{d t^{2}} \bar{A}_{2}\left(G_{i}^{(k)}\right)\left[\left\{\theta_{l, s}^{\text {ext }}\right\} ; t=0\right]=\sum_{s=1}^{4} \int\left\{\left[\prod_{s} d \theta_{1, s}\right]\left(C_{j_{1}}\left(\left\{\theta_{1, u}^{\text {ext }}\right\} ;\left\{\theta_{1, u}^{0}\right\}\right) \partial_{\theta ; s}^{2} C_{j_{2}}\left(\left\{\theta_{2, v}^{\text {ext }}\right\} ;\left\{\theta_{1, v}^{0}\right\}\right)\right)\right. \\
& \left.\times \int\left[\prod_{\ell \neq 1} d \tilde{\theta}_{\ell, s}\right]\left[\left(\tilde{\theta}_{2, s}^{0}\right)^{2} \prod_{\ell \neq 1} C_{i_{\ell}}\left(\left\{\tilde{\theta}_{\ell(v), u}\right\} ;\left\{\tilde{\theta}_{\ell\left(v^{\prime}\right), u}\right\}\right)\right] \prod_{v \in \mathcal{V}} \delta\left(\tilde{\theta}_{v, s}-\tilde{\theta}_{v, s^{\prime}}\right)\right\} .
\end{aligned}
$$

In fact, the internal part does not depend on the strand index $s$ because, from the beginning, all $s=1,2,3,4$ are treated in a symmetric manner. Hence all integrations as $\int d \tilde{\theta}_{2, s}^{0}$, for any $s$, should produce the same result. Moreover, the $\tilde{\theta}_{2, s}^{0}$ factors are of order $M^{-2 i_{2}}$. This contribution cancels the internal quadratic divergence (same as for the mass local part) thus yielding a logarithmic divergence for a wave function renormalization.

Lemma 12. The remainder $R_{2}$ of the amplitude interpolation can be bounded by

$$
\left|R_{2}\right| \leq K M^{-3\left(i\left(G_{i}^{(k)}\right)-e\left(G_{i}^{(k)}\right)\right)} M^{\omega\left(G_{i}^{(k)}\right)}, \quad e\left(G_{i}^{(k)}\right)=\sup _{\text {lexternal to } G_{i}^{(k)}} j_{l}, \quad i\left(G_{i}^{(k)}\right)=\inf _{l \in G_{i}^{(k)}} i_{l},
$$

for some constant $K$.

Proof. As in the earlier setting, in the convenient variables, the $T^{3}$ operator applied on the propagators yields a prefactor of the form $\left|\left(\theta_{2, s_{k}}^{0}-\theta_{1, s_{k}}^{0}\right)^{3}\right|$ which can be bounded by $M^{-3 i\left(G_{i}^{(k)}\right)}$ whereas each derivative $\partial_{\theta ; s} C_{j_{l}}$ yields a good factor $M^{e\left(G_{i}^{(k)}\right)}$ according 24 . We infer the bound (removing the differences $t\left(\theta_{l, s}^{0}-\theta_{l^{\prime}, s}^{0}\right)$ due to strong internal decay)

$$
\begin{aligned}
\left|R_{2}\right| \leq & K M^{-3\left(i\left(G_{i}^{(k)}\right)-e\left(G_{i}^{(k)}\right)\right)} \int\left[\prod_{\ell} d \theta_{\ell, s}\right]\{ \\
& \left(\sum_{l=1}^{6}\left[\prod_{q \neq l} M^{2 j_{q}} e^{\left.-\delta M^{j_{q}} \sum_{s} \mid \theta_{q, s}^{\mathrm{ext}}-\theta_{q^{\prime}\left(v^{\prime}\right), s}^{0}\right]} M^{2 e\left(G_{i}^{(k)}\right)} e^{-\delta M^{j_{l}} \sum_{s}\left|\theta_{l, s}^{\mathrm{ext}}-\theta_{l^{\prime}\left(v^{\prime}\right), s}^{0}\right|}\right)\right.
\end{aligned}
$$




$$
\left.\left[\prod_{\ell \neq l} C_{i_{\ell}}\left(\left\{\theta_{\ell(v), s}\right\} ;\left\{\theta_{\ell\left(v^{\prime}\right), s}\right\}\right)\right] \prod_{v \in \mathcal{V}} \delta\left(\theta_{v, s}-\theta_{v, s^{\prime}}\right)\right\} .
$$

In summary, the expansion of the two-point function gives a local contribution to the mass which is quadratically divergent, a wave function renormalization which is logarithmically divergent and a remainder which will allow to sum on the momentum assignments.

The fact that the theory is renormalizable at all order of perturbations then follows from the standard techniques of summation on momentum assignments developed in [55]. Remark that the theory is well-prepared by the multiscale expansion to be written in terms of an infinite set of effective couplings which follow the renormalization group trajectory. It is almost a pity to add the counterterms corresponding to non quasi-local subgraphs to reexpress the theory in terms of the standard renormalized couplings. This does not of course introduce any divergence and the coefficients of that renormalized power series can be proved term by term finite. However the renormalized series is in fact much less natural than the effective one, and is plagued by large undesirable contributions called renormalons. These phenomena, analyzed at length in [55], will not be further discussed here.

\section{Conclusion}

The tensor model presented here is not claimed to be the right final model for quantum gravity but hopefully a first step in that direction.

It also completes nicely the progressive discovery of new forms of renormalization group with different types of divergent graphs. There seems to be a natural hierarchy of these forms. In ordinary just renormalizable models such as the local $\phi_{4}^{4}$ theory or Yang-Mills theory, the divergence degree is simply a function of the number of external legs. In the condensed matter theory of interacting electrons in any dimension, the renormalization group is already very different. It is governed by the approach to the Fermi surface, which is a codimension 2 singularity in space-time. In this particular instance, the important flow is that of the Cooper pair four-point coupling constant in the $s=0$ channel. Only a very simple category of four-point graphs contribute to that flow, namely those which are chains of bubbles. They are also the ones leading in the $1 / N$ expansion for vector models and form a geometric series. The BCS transition can be analyzed accordingly. $N$ can be interpreted as the number of sectors or quasi particles around the Fermi surface [63]. We propose to consider the renormalization group for such models as vector-like.

In the Grosse-Wulkenhaar model [51, 52] as well as the $\phi_{\star}^{6}$-theory as developed in [64], the non-commutativity of the underlying space-time translates into a matrix representation of the theory with a perturbative expansion indexed by ribbon graphs. The divergent graphs are the planar graphs with all external legs incident on a single face. They are the ones leading the $1 / N$ matrix expansion. Obviously the renormalization group for such models should be called matrix-like.

The models of this paper pioneers a new category of renormalization group, based on tensor fields of rank higher than 2. The key divergent graphs are the melonic ones. Their renormalization group should be called of tensor-type. This issue and the systematic study of such models including their renormalization group flows, symmetries (either in the spirit 
of [65, 66] or in that of [47]), the issue of their constructive stability and possible phase transitions is left to future studies (the corresponding program and its relationship to other approaches to quantum gravity is further discussed in [67]).

As a final remark, we conjecture that if we restrict the couplings of the model studied above to the precise values given by the integration of the standard colored theory, that is if we link $\lambda_{6 ; 1} \lambda_{6 ; 2}$ and $\lambda_{4 ; 1}$ as they should be when integrating the $D$ fields of a colored tensor theory with single coupling $\lambda$, we should obtain an even more interesting just renormalizable theory with a single coupling, i.e. the corresponding manifold should be stable under the renormalization group flow.

\section{Appendix}

\section{A Propagator bounds}

We consider the propagator in the slice $i$ as

$$
\begin{aligned}
C_{i}\left(\left\{\theta_{s}\right\} ;\left\{\theta_{s}^{\prime}\right\}\right) & =k \int_{M^{-2(i+1)}}^{M^{-2 i}} \frac{e^{-m^{2} \alpha}}{\alpha^{2}} e^{-\frac{1}{4 \alpha} \sum_{s}\left[\theta_{s}-\theta_{s}^{\prime}\right]^{2}} T\left(\alpha ;\left\{\theta_{s}\right\} ;\left\{\theta_{s}^{\prime}\right\}\right) d \alpha, \\
T\left(\alpha ;\left\{\theta_{s}\right\} ;\left\{\theta_{s}^{\prime}\right\}\right) & =\prod_{s=1}^{4}\left\{1+2 \sum_{n=1}^{\infty} e^{-\frac{\pi^{2} n^{2}}{\alpha}} \cosh \left[\frac{n \pi}{\alpha}\left[\theta_{s}-\theta_{s}^{\prime}\right]\right]\right\} .
\end{aligned}
$$

Since only positive terms are involved in each series in the product $T$, we can find an integral bounding the series as

$$
\sum_{n=1}^{\infty} e^{-\frac{\pi^{2} n^{2}}{\alpha}} \cosh \left[\frac{n \pi}{\alpha} \Theta\right] \leq \int_{1}^{\infty} e^{-\frac{\pi^{2} x^{2}}{\alpha}} \cosh \left[\frac{\pi \Theta}{\alpha} x\right] d x
$$

where $|\Theta|<2 \pi$. The latter integral can be recast in terms of Gaussian error functions:

$$
\begin{aligned}
& \int_{1}^{\infty} e^{-\frac{\pi^{2} x^{2}}{\alpha}} \cosh \left[\frac{\pi \Theta}{\alpha}\right] d x=\frac{\sqrt{\alpha} e^{\frac{\Theta^{2}}{4 \alpha}}}{4 \sqrt{\pi}}\left(\operatorname{erfc}\left(\frac{2 \pi-\Theta}{2 \sqrt{\alpha}}\right)+\operatorname{erfc}\left(\frac{2 \pi+\Theta}{2 \sqrt{\alpha}}\right)\right), \\
& \operatorname{erfc}(z)=1-\frac{2}{\sqrt{\pi}} \int_{0}^{z} e^{-t^{2}} d t, \quad z \in \mathbb{C} ; \quad \operatorname{erfc}(x) \leq \frac{2}{\sqrt{\pi}} \frac{e^{-x^{2}}}{x+\sqrt{x^{2}+\frac{4}{\pi}}} \leq e^{-x^{2}}, \quad x>0,
\end{aligned}
$$

therefore, given $-2 \pi<\Theta<2 \pi$,

$$
\begin{aligned}
& e^{\frac{\Theta^{2}}{4 \alpha}}\left(\operatorname{erfc}\left(\frac{2 \pi-\Theta}{2 \sqrt{\alpha}}\right)+\operatorname{erfc}\left(\frac{2 \pi+\Theta}{2 \sqrt{\alpha}}\right)\right) \leq e^{\frac{\Theta^{2}}{4 \alpha}}\left[e^{-\left(\frac{2 \pi-\Theta}{2 \sqrt{\alpha}}\right)^{2}}+e^{-\left(\frac{2 \pi+\Theta}{2 \sqrt{\alpha}}\right)^{2}}\right] \\
& \leq e^{\frac{-\pi^{2}}{\alpha}}\left[e^{\frac{\pi \Theta}{2 \alpha}}+e^{\frac{-\pi \Theta}{2 \alpha}}\right] \leq 2 .
\end{aligned}
$$

Then, combining (A.4), A.3 and A.2, we find a bound for $T$ (A.1) as

$$
T\left(\alpha ;\left\{\theta_{s}\right\} ;\left\{\theta_{s}^{\prime}\right\}\right) \leq \prod_{s=1}^{4}\left\{1+\frac{\sqrt{\alpha}}{\sqrt{\pi}}\right\}
$$


hence the following bound is achieved

$$
C_{i}\left(\left\{\theta_{s}\right\} ;\left\{\theta_{s}^{\prime}\right\}\right) \leq k \int_{M^{-2(i+1)}}^{M^{-2 i}} \frac{e^{-m^{2} \alpha}}{\alpha^{2}} e^{-\frac{1}{4 \alpha} \sum_{s}\left[\theta_{s}-\theta_{s}^{\prime}\right]^{2}} \prod_{s=1}^{4}\left\{1+\frac{\sqrt{\alpha}}{\sqrt{\pi}}\right\} d \alpha
$$

By expanding the product, it can be observed that the term with coefficient 1 is the dominant one. The sum of remaining terms, including powers of $\sqrt{\alpha}$ in their numerator, can be bounded by a constant (mainly, the number of terms) times the leading term. Indeed, for instance, focusing on the subleading term of the form

$$
\begin{aligned}
k \int_{M^{-2(i+1)}}^{M^{-2 i}} \frac{e^{-m^{2} \alpha}}{\alpha^{2}} e^{-\frac{1}{4 \alpha} \sum_{s}\left[\theta_{s}-\theta_{s}^{\prime}\right]^{2}} \frac{\sqrt{\alpha}}{\sqrt{\pi}} d \alpha & =k^{\prime} \int_{M^{-2(i+1)}}^{M^{-2 i}} \frac{e^{-m^{2} \alpha}}{\alpha^{\frac{3}{2}}} e^{-\frac{1}{4 \alpha} \sum_{s}\left[\theta_{s}-\theta_{s}^{\prime}\right]^{2}} d \alpha \\
& \leq k^{\prime} \int_{M^{-2(i+1)}}^{M^{-2 i}} \frac{e^{-m^{2} \alpha}}{\alpha^{2}} e^{-\frac{1}{4 \alpha} \sum_{s}\left[\theta_{s}-\theta_{s}^{\prime}\right]^{2}} .
\end{aligned}
$$

Higher order terms involve $\alpha^{\frac{d}{2}}, d \geq 1$, in the numerator, hence they will be less divergent. This validates the bound $C_{i}(25)$ for all $i \gg 1$.

For the last slice, we have

$$
C_{0}\left(\left\{\theta_{s}\right\} ;\left\{\theta_{s}^{\prime}\right\}\right) \leq k \int_{1}^{\infty} \frac{e^{-m^{2} \alpha}}{\alpha^{2}} e^{-\frac{1}{4 \alpha} \sum_{s}\left[\theta_{s}-\theta_{s}^{\prime}\right]^{2}} \prod_{s=1}^{4}\left\{1+\frac{\sqrt{\alpha}}{\sqrt{\pi}}\right\} d \alpha .
$$

This expression can be bounded, this time, by the term containing the highest power of $\sqrt{\alpha}$ :

$$
\begin{aligned}
C_{0}\left(\left\{\theta_{s}\right\} ;\left\{\theta_{s}^{\prime}\right\}\right) & \leq K^{\prime} \int_{1}^{\infty} \frac{e^{-m^{2} \alpha}}{\alpha^{2}} e^{-\frac{1}{4 \alpha} \sum_{s}\left[\theta_{s}-\theta_{s}^{\prime}\right]^{2}} \alpha^{2} d \alpha \\
& \leq K^{\prime} \sup _{\alpha \in[1,+\infty)}\left(e^{-m^{2} \alpha / 2} \alpha^{2} e^{-\frac{1}{4 \alpha} \sum_{s}\left[\theta_{s}-\theta_{s}^{\prime}\right]^{2}}\right) \int_{1}^{\infty} \frac{e^{-m^{2} \alpha / 2}}{\alpha^{2}} d \alpha \\
& \leq K^{\prime \prime} \int_{1}^{\infty} \frac{e^{-m^{2} \alpha / 2}}{\alpha^{2}} d \alpha
\end{aligned}
$$

which validates 27 .

\section{B The Three Dimensional Case}

In three dimensions, there is also a just renormalizable similar model but with propagator $\left(\sum_{s=1}^{3}\left|p_{s}\right|+m\right)^{-1}$ and a single melonic "pillow" interaction

$$
S_{4}=\int_{h_{j}} \psi_{1,2,3} \bar{\psi}_{1^{\prime}, 2,3} \psi_{1^{\prime}, 2^{\prime}, 3^{\prime}} \bar{\psi}_{1,2^{\prime}, 3^{\prime}}+\text { permutations }
$$

As usual, we have to introduce a mass counterterm $V_{2}$ and a $V_{2}^{\prime}$ wave function $\sum_{s=1}^{3}\left|p_{s}\right|$ counterterm.

The scaling of the sliced propagator is now

$$
C^{i} \leq K M^{2 i} e^{-\delta M^{i} \sum_{s}\left|\theta_{s}-\theta_{s}^{\prime}\right|}
$$


hence the power counting is $\omega_{d}=-L+F+V_{2}^{\prime}$. Following the analysis of Section 4 and 5 , with same notations we have $4 V_{4}+2\left(V_{2}+V_{2}^{\prime}\right)=2 L+N_{\text {ext }}$ and

$$
V_{\mathcal{G}_{\text {color }}}=4 V_{4}+2\left(V_{2}+V_{2}^{\prime}\right), \quad L_{\mathcal{G}_{\text {color }}}=L+L_{\text {int } ; \mathcal{G}_{\text {color }}}=\frac{1}{2}\left(4 V_{\mathcal{G}_{\text {color }}}-N_{\text {ext }}\right)
$$

There are 3 jackets in $\mathcal{G}_{\text {color }}$. Each face of the graph $\mathcal{G}_{\text {color }}$ (open or closed) is shared by exactly 2 jackets so that $\sum_{J} F_{J}=2 F_{\mathcal{G}_{\text {color }}}$ and

$$
\sum_{J}\left(F_{\text {int } ; \widetilde{J} ; \mathcal{G}}+F_{\text {int } ; \widetilde{J} ; \mathcal{G}_{\text {color }}}+F_{\text {ext } ; \widetilde{J}}\right)=2 F_{\text {int } ; \mathcal{G}}+2 F_{\text {int } ; \mathcal{G}_{\text {color }}}+\sum_{J} F_{\text {ext } ; \widetilde{J}}
$$

Each $\varphi^{4}$ vertex contains 4 internal faces and each $\varphi^{2}$ vertex contains 3 internal faces so that $F_{\text {int } ; \mathcal{G}_{\text {color }}}=4 V_{4}+3\left(V_{2}+V_{2}^{\prime}\right)$. Hence

$$
\sum_{J}\left[-V_{J}+L_{J}\right]=3\left[4 V_{4}+2\left(V_{2}+V_{2}^{\prime}\right)\right]-\frac{3}{2} N_{\mathrm{ext}},
$$

and

$$
F_{\text {int } ; \mathcal{G}}=2 V_{4}-\frac{3}{4} N_{\text {ext }}+3-\sum_{J} g_{\widetilde{J}}-\frac{1}{2} \sum_{J} F_{\text {ext } ; \widetilde{J}} .
$$

The boundary graph is a closed ribbon graph living in dimension $D-1=2$, hence has a single jacket. Since each external leg of the initial graph $\mathcal{G}$ has 3 strands and an external leg is made with two end-points belonging to two external legs, we have in this simpler case

$$
L_{\partial \mathcal{G}}-V_{\partial \mathcal{G}}=\frac{1}{2} N_{\mathrm{ext}}, F_{\partial \mathcal{G}}=2\left(C_{\partial \mathcal{G}}-1\right)-2 g_{\partial \mathcal{G}}+2+\frac{1}{2} N_{\mathrm{ext}}, \sum_{J} F_{\mathrm{ext} ; \widetilde{J}}=F_{\partial \mathcal{G}}
$$

(with again $g_{\partial \mathcal{G}}=\sum_{\rho} g_{\partial \mathcal{G}_{\rho}}$ ) and finally we get the divergence degree

$$
\omega_{d}(\mathcal{G})=-V_{2}-\frac{1}{2}\left(N_{\mathrm{ext}}-4\right)-\sum_{J} g_{\widetilde{J}}+g_{\partial \mathcal{G}}-\left(C_{\partial \mathcal{G}}-1\right),
$$

appearing as a simpler analog of Theorem 2 and formula (42). The complete proof of the renormalizability of this model following Section 5 and 6 has been addressed in a recent ${ }^{11}$ work [68].

\section{Acknowledgements}

We thank R. Gurau for useful discussions at various stages of this work and for a critical reading of the manuscript. The authors also thank the referee for his careful reading and interesting remarks improving Lemma 4 and the presentation of this paper.

Research at Perimeter Institute is supported by the Government of Canada through Industry Canada and by the Province of Ontario through the Ministry of Research and Innovation.

\footnotetext{
${ }^{11}$ The said manuscript was published shortly after the publication of the first version of this paper.
} 


\section{References}

[1] V. Rivasseau, "Towards Renormalizing Group Field Theory," PoS C NCFG2010, 004 (2010) arXiv:1103.1900 [gr-qc]].

[2] P. Di Francesco, P. H. Ginsparg and J. Zinn-Justin, "2-D Gravity and random matrices," Phys. Rept. 254, 1 (1995) arXiv:hep-th/9306153.

[3] F. David, "A Model Of Random Surfaces With Nontrivial Critical Behavior," Nucl. Phys. B 257, 543 (1985).

[4] V. A. Kazakov, "Bilocal regularization of models of random surfaces," Phys. Lett. B 150, $282(1985)$.

[5] E. Brézin and V. A. Kazakov, "Exactly solvable field theories of closed strings," Phys. Lett. B 236, 144 (1990).

[6] M. R. Douglas and S. H. Shenker, "Strings in less than one dimension," Nucl. Phys. B 335, 635 (1990).

[7] D. J. Gross and A. A. Migdal, "Nonperturbative two-dimensional quantum gravity," Phys. Rev. Lett. 64, 127 (1990).

[8] V. G. Knizhnik, A. M. Polyakov and A. B. Zamolodchikov, "Fractal structure of 2d quantum gravity," Mod. Phys. Lett. A 3, 819 (1988).

[9] F. David, "Conformal field theories coupled to 2d gravity in the conformal gauge," Mod. Phys. Lett. A 3, 1651 (1988).

[10] J. Distler and H. Kawai, "Conformal field theory and 2d quantum gravity or who's afraid of Joseph Liouville?," Nucl. Phys. B321, 509 (1989).

[11] B. Duplantier, "Conformal random geometry," in Les Houches, Session LXXXIII: Mathematical Statistical Physics, (July, 2005), Editors A. Bovier, F. Dunlop, F. den Hollander, A. van Enter and J. Dalibard, Elsevier, 2006, 101-217, math-ph/0608053.

[12] G. 't Hooft, "A Planar Diagram theory for Strong Interactions," Nucl. Phys. B 72, 461 (1974).

[13] J. Ambjorn, B. Durhuus and T. Jonsson, "Three-Dimensional Simplicial Quantum Gravity And Generalized Matrix Models," Mod. Phys. Lett. A 6, 1133 (1991).

[14] M. Gross, "Tensor models and simplicial quantum gravity in > 2-D," Nucl. Phys. Proc. Suppl. 25A, 144 (1992).

[15] N. Sasakura, "Tensor model for gravity and orientability of manifold," Mod. Phys. Lett. A 6, 2613 (1991).

[16] J. Ambjorn and S. Varsted, "Three-dimensional simplicial quantum gravity," Nucl. Phys. B 373, 557 (1992). 
[17] N. Sasakura, "Canonical tensor models with local time," arXiv:1111.2790 [hep-th]].

[18] N. Sasakura, "Tensor models and hierarchy of n-ary algebras," Int. J. Mod. Phys. A26, 3249-3258 (2011) arXiv:1104.5312 [hep-th]].

[19] S. -J. Rey and F. Sugino, "A Nonperturbative Proposal for Nonabelian Tensor Gauge Theory and Dynamical Quantum Yang-Baxter Maps," arXiv:1002.4636 [hep-th]].

[20] D. V. Boulatov, "A Model of three-dimensional lattice gravity," Mod. Phys. Lett. A 7, 1629 (1992) arXiv:hep-th/9202074.

[21] H. Ooguri, "Topological lattice models in four-dimensions," Mod. Phys. Lett. A 7, 2799 (1992) arXiv:hep-th/9205090.

[22] L. Freidel, "Group field theory: An overview," Int. J. Theor. Phys. 44, 1769 (2005) arXiv:hep-th/0505016.

[23] D. Oriti, "The group field theory approach to quantum gravity: some recent results," arXiv:0912.2441 [hep-th]].

[24] D. Oriti, "The microscopic dynamics of quantum space as a group field theory," arXiv:1110.5606 [hep-th].

[25] J. W. Barrett and L. Crane, "An Algebraic interpretation of the Wheeler-DeWitt equation," Class. Quant. Grav. 14, 2113 (1997) gr-qc/9609030.

[26] J. Engle, E. Livine, R. Pereira and C. Rovelli, "LQG vertex with finite Immirzi parameter," Nucl. Phys. B799, 136 (2008) arXiv:0711.0146 [gr-qc]].

[27] L. Freidel and K. Krasnov, "A New Spin Foam Model for 4d Gravity," Class. Quant. Grav. 25, 125018 (2008) arXiv:0708.1595 [gr-qc]].

[28] J. Ben Geloun, R. Gurau and V. Rivasseau, "EPRL/FK Group Field Theory," Europhys. Lett. 92, 60008 (2010) arXiv:1008.0354 [hep-th]].

[29] T. Krajewski, J. Magnen, V. Rivasseau, A. Tanasa and P. Vitale, "Quantum Corrections in the Group Field Theory Formulation of the EPRL/FK Models," Phys. Rev. D 82, 124069 (2010) arXiv:1007.3150 [gr-qc]].

[30] L. Freidel, R. Gurau and D. Oriti, "Group field theory renormalization - the 3d case: power counting of divergences," Phys. Rev. D 80, 044007 (2009) arXiv:0905.3772 [hepth]].

[31] J. Magnen, K. Noui, V. Rivasseau and M. Smerlak, "Scaling behavior of threedimensional group field theory," Class. Quant. Grav. 26, 185012 (2009) arXiv:0906.5477 [hep-th]].

[32] J. Ben Geloun, J. Magnen and V. Rivasseau, "Bosonic Colored Group Field Theory," Eur. Phys. J. C 70, 1119 (2010) arXiv:0911.1719 [hep-th]]. 
[33] J. Ben Geloun, T. Krajewski, J. Magnen and V. Rivasseau, "Linearized Group Field Theory and Power Counting Theorems," Class. Quant. Grav. 27, 155012 (2010) arXiv:1002.3592 [hep-th]].

[34] V. Bonzom and M. Smerlak, "Bubble divergences from cellular cohomology," Lett. Math. Phys. 93, 295 (2010) [arXiv:1004.5196 [gr-qc]].

[35] V. Bonzom and M. Smerlak, "Bubble divergences from twisted cohomology," arXiv:1008.1476 [math-ph].

[36] V. Bonzom and M. Smerlak, "Bubble divergences: sorting out topology from cell structure," arXiv:1103.3961 [gr-qc].

[37] R. Gurau, "Colored Group Field Theory," Commun. Math. Phys. 304, 69 (2011) arXiv:0907.2582 [hep-th]].

[38] R. Gurau, "The 1/N expansion of colored tensor models," Annales Henri Poincare 12, 829-847 (2011). arXiv:1011.2726 [gr-qc]].

[39] R. Gurau and V. Rivasseau, "The 1/N expansion of colored tensor models in arbitrary dimension," Europhys. Lett. 95, 50004 (2011). [arXiv:1101.4182 [gr-qc]].

[40] R. Gurau, "The complete $1 / \mathrm{N}$ expansion of colored tensor models in arbitrary dimension," arXiv:1102.5759 [gr-qc].

[41] V. Bonzom, R. Gurau, A. Riello and V. Rivasseau, "Critical behavior of colored tensor models in the large N limit," Nucl. Phys. B 853, 174 (2011) [arXiv:1105.3122 [hep-th]].

[42] V. Bonzom, R. Gurau and V. Rivasseau, "The Ising Model on Random Lattices in Arbitrary Dimensions," arXiv:1108.6269 [hep-th].

[43] D. Benedetti and R. Gurau, "Phase Transition in Dually Weighted Colored Tensor Models," Nucl. Phys. B 855, 420 (2012) [arXiv:1108.5389 [hep-th]].

[44] R. Gurau and J. P. Ryan, "Colored Tensor Models - a review," arXiv:1109.4812 [hep-th].

[45] R. Gurau, "Topological Graph Polynomials in Colored Group Field Theory," Annales Henri Poincare 11, 565 (2010) arXiv:0911.1945 [hep-th]].

[46] R. Gurau, "Lost in Translation: Topological Singularities in Group Field Theory," Class. Quant. Grav. 27, 235023 (2010) arXiv:1006.0714 [hep-th]].

[47] A. Baratin, F. Girelli and D. Oriti, "Diffeomorphisms in group field theories," Phys. Rev. D 83, 104051 (2011) arXiv:1101.0590 [hep-th]].

[48] R. Gurau, "The Double Scaling Limit in Arbitrary Dimensions: A Toy Model," arXiv:1110.2460 [hep-th].

[49] R. Gurau, "A generalization of the Virasoro algebra to arbitrary dimensions," Nucl. Phys. B 852, 592 (2011) arXiv:1105.6072 [hep-th]]. 
[50] R. Gurau, "Universality for Random Tensors," arXiv:1111.0519 [math.PR].

[51] H. Grosse and R. Wulkenhaar, "Renormalisation of phi**4 theory on noncommutative $\mathrm{R}^{* *} 4$ in the matrix base," Commun. Math. Phys. 256, 305 (2005) arXiv:hepth/0401128.

[52] V. Rivasseau, F. Vignes-Tourneret and R. Wulkenhaar, "Renormalization of noncommutative phi**4-theory by multi-scale analysis," Commun. Math. Phys. 262, 565 (2006) arXiv:hep-th/0501036].

[53] D. Oriti and L. Sindoni, "Towards classical geometrodynamics from Group Field Theory hydrodynamics," New J. Phys. 13, 025006 (2011) [arXiv:1010.5149 [gr-qc]].

[54] J. Ben Geloun and V. Bonzom, "Radiative corrections in the Boulatov-Ooguri tensor model: The 2-point function," Int. J. Theor. Phys. 50, 2819 (2011) arXiv:1101.4294 [hep-th]].

[55] V. Rivasseau, "From perturbative to constructive renormalization," Princeton series in physics (Princeton Univ. Pr., Princeton, 1991).

[56] "Handbook of Mathematical Functions," 10th edition Appl. Math. Ser. 55, Section 19, A. Abramowitz and I. A. Stegun editors (Dover, NY, 1972).

[57] G. Gallavotti and F. Nicolo, "Renormalization theory in four-dimensional scalar fields. I," Commun. Math. Phys. 100, 545 (1985).

[58] V. Rivasseau, "Non-commutative renormalization," arXiv:0705.0705 [hep-th].

[59] S. Lins, "Gems, Computers and Attractors for 3-Manifolds," Series on Knots and Everything, Vol. 5 (World Scientific, Singapore, 1995).

[60] M. Ferri and C. Gagliardi, "Cristallisation moves," Pacific Journal of Mathematics 100, 85-103 (1982).

[61] T. Filk, "Divergencies in a field theory on quantum space," Phys. Lett. B 376 (1996) 53-58.

[62] R. Gurau, J. Magnen, V. Rivasseau and F. Vignes-Tourneret, "Renormalization of noncommutative phi**4(4) field theory in x space," Commun. Math. Phys. 267, 515 (2006) arXiv:hep-th/0512271].

[63] J. Feldman, J. Magnen, V. Rivasseau and E. Trubowitz, "An Intrinsic 1/N expansion for many fermion systems," Europhys. Lett. 24, 437 (1993).

[64] Z. Wang and S. Wan, "Renormalization of Orientable Non-Commutative Complex Phi**6(3) Model," Annales Henri Poincare 9, 65 (2008) [arXiv:0710.2652 [hep-th]].

[65] J. Ben Geloun, "Classical Group Field Theory," arXiv:1107.3122 [hep-th]]. 
[66] J. Ben Geloun, "Ward-Takahashi identities for the colored Boulatov model," J. Phys. A 44, 415402 (2011) arXiv:1106.1847 [hep-th]].

[67] V. Rivasseau, "Quantum Gravity and Renormalization: The Tensor Track," arXiv:1112.5104 [hep-th].

[68] J. Ben Geloun and D. O. Samary, "3D Tensor Field Theory: Renormalization and One-loop $\beta$-functions," arXiv:1201.0176 [hep-th]. 